\title{
UNA HERRAMIENTA TIC PARA LA REDACCIÓN DEL TRABAJO DE FIN DE GRADO (TFG)
}

\author{
AN ICT TOOL FOR THE WRITING OF THE FINAL DEGREE PROJECT (TFG)
}

\author{
IRIA DA CUNHA \\ Universidad Nacional de Educación a Distancia (UNED) \\ iriad@flog.uned.es \\ https://orcid.org/0000-0002-7707-1574
}

Recibido: $15 / 01 / 2020$

Aceptado: 01/05/2020

\begin{abstract}
Resumen
Desde la implantación del Espacio Europeo de Educación Superior a través del Plan Bolonia, en la universidad española es obligatorio que los estudiantes elaboren un Trabajo de Fin de Grado (TFG). El objetivo del presente trabajo es desarrollar una herramienta TIC (Tecnologías de la Información y la Comunicación) para ayudar a los estudiantes en la redacción del TFG en español. La herramienta tiene forma de editor de textos en línea e integra recursos de Procesamiento del Lenguaje Natural (PLN), como un analizador morfológico y un segmentador discursivo. Está dividida en tres módulos, que ayudan al estudiante a: 1) estructurar el TFG, 2) darle formato y corregirlo ortográficamente, y 3) revisar la adecuación del texto, mediante recomendaciones lingüísticas específicas para este género textual, relacionadas, por ejemplo, con oraciones largas, siglas, definiciones o unidades léxicas subjetivas. La evaluación user-driven realizada con estudiantes universitarios ofrece resultados positivos del uso de la herramienta.
\end{abstract}

\begin{abstract}
Since the implementation of the European Higher Education Area through the Bologna Plan, in the Spanish university it is mandatory for students to prepare a Final Degree Project (TFG). The aim of this work is to develop an ICT (Information and Communication Technologies) tool to help students in the writing of the TFG in Spanish. The tool is an online text editor and integrates Natural Language Processing (NLP) resources, such as a Part of Speech tagger and a discourse segmenter. It is divided into three modules, which help the student to: 1) structure the TFG, 2) format the text and correct its spelling, and 3) review the adequacy of the text, through specific linguistic recommendations for this textual genre, related, for example, with long sentences, initialisms, definitions or subjective lexical units. The userdriven evaluation carried out with university students shows positive results.
\end{abstract}

Para citar este artículo / To cite this article: da Cunha, Iria (2020). Una herramienta TIC para la redacción del Trabajo de Fin de Grado (TFG). ELUA, 34: 39-72. https://doi.org/10.14198/ELUA2020.34.2

Enlace / Link: https://oi.org/10.14198/ELUA2020.34.2 
PALABRAS CLAVE: herramienta TIC (Tecnologías de la Información y la Comunicación), Trabajo de Fin de Grado (TFG), textos académicos, redacción, Procesamiento del Lenguaje Natural (PLN).
KEYWORDS: ICT (Information and Communication Technologies) Tool, Final Degree Project (TFG), Academic Texts, Writing, Natural Language Processing (NLP).

\section{INTRODUCCIÓN}

Con la implantación del nuevo Espacio Europeo de Educación Superior a través del Plan Bolonia, desde 2010 es obligatorio que los estudiantes de todas las universidades españolas elaboren un Trabajo de Fin de Grado (TFG) para poder finalizar sus estudios superiores de grado. Sin embargo, los estudiantes universitarios no están familiarizados con muchos de los géneros textuales utilizados en el contexto académico y, precisamente, el TFG es uno de ellos. Como subraya Núñez (2015: 17):

Pese a que es común la idea de que los estudiantes ingresan en los estudios superiores con un déficit en esta competencia [comunicativa], los problemas y dificultades de comunicación que tienen y a los que se enfrentan no solo se deben a estas carencias. [...] Los textos que leen y escriben los estudiantes tienen características particulares que difieren, en ocasiones, de aquellos con los que ya estaban familiarizados. Así, las formas de comunicación de las comunidades discursivas académicas son singulares y los estudiantes no están experimentados con ellas, por lo que encuentran, en ocasiones, una dificultad que les parece imposible.

Realizar un trabajo de la envergadura de un TFG no es una tarea sencilla y, para que puedan superarla con éxito, es necesario ofrecer a los estudiantes recursos y herramientas específicos para ello. Tal como indica Cabré (2016: 11):

Una de las tareas importantes que los estudiantes universitarios deben aprender a realizar en el ámbito académico es la investigación. Ninguna carrera de estudio puede permitirse que sus estudiantes acaben sin haber realizado un proyecto. Para ello, los profesores deben proporcionarles las herramientas necesarias para llevarlo a cabo.

No obstante, la realidad es que, por el momento, por lo general y salvo excepciones, en las distintas universidades españolas no se prepara en profundidad a los estudiantes para la redacción del TFG, sea cual sea su especialidad. Tal como indica Sánchez-Jiménez (2018: 304), resulta contradictorio que precisamente los estudiantes sean (parcialmente) evaluados a través de un género textual al cual es la primera vez que se enfrentan en muchos casos y sobre el cual no se les ha dado una formación exhaustiva:

Al contrario de lo que comúnmente se piensa (véase Kruse, 2003), los estudiantes no han sido suficientemente preparados en los niveles de enseñanza previos para hacer frente a las necesidades expresivas que se les exige conocer en la universidad, las cuales, por otro lado, requieren nuevas competencias de lectura y escritura no fácilmente transferibles (Castelló y otros, 2012). Por este motivo, resulta particularmente contradictorio que a partir de la entrada en vigor del Plan Bolonia los estudiantes sean evaluados mediante la revisión de sus productos escritos, para cuya elaboración específica no han recibido una formación 
apropiada, a diferencia de lo que sí ha sucedido en otros países de los ámbitos anglosajón e hispanoamericano desde hace ya varias décadas (véanse Carlino, 2005; Castelló, 2014; Sánchez-Jiménez, 2016). Una consecuencia de este hecho ha sido la revisión curricular de los programas de grado, los cuales comienzan a incluir asignaturas centradas en la escritura académica entre sus cursos, junto a la creación de centros de escritura ubicados en algunas universidades españolas (Martínez Pasamar y Llamas Saíz, 2015).

La elaboración de un TFG se sitúa en el contexto de la comunicación especializada. Tal como explica Cabré (1992, 1999), la comunicación especializada tiene ciertas particularidades que la distinguen de la comunicación general, según los elementos del esquema comunicativo de Jakobson (1963). Estas particularidades se refieren principalmente a tres elementos: el emisor, el mensaje y el receptor. En primer lugar, el emisor ha de ser un especialista del ámbito especializado sobre el que trata el texto, como por ejemplo la medicina, la economía, la lingüística, la informática, la química, el derecho, el medio ambiente, etc. En el caso del TFG, el estudiante debe situarse en el lugar del especialista como emisor. En segundo lugar, en el caso de la comunicación especializada, el mensaje es el mismo texto especializado (ya sea oral o escrito) que se transmite. El estudiante no debe olvidar que un trabajo académico es un producto textual que debe cumplir los requisitos que se esperan de este tipo de textos especializados. En tercer lugar, en el contexto de la comunicación especializada, el receptor puede ser de naturaleza diversa. Concretamente, suelen mencionarse tres tipos de receptores posibles: a) otros especialistas del ámbito especializado del que trata el texto, b) estudiantes o aprendices interesados en aprender sobre dicho ámbito, y c) el público general o lego en la materia, que simplemente tiene un interés puntual o esporádico sobre el ámbito, pero no tiene formación sobre él. En el caso del TFG, los receptores principales serán otros especialistas del mismo ámbito (principalmente los directores del trabajo, los miembros del tribunal que lo evaluará y otros especialistas que investiguen sobre temas afines al del trabajo), y estudiantes que se encuentren en la fase de elaboración de su propio TFG y necesiten adquirir información sobre dicho tema o ver otros trabajos para tomar como modelo.

Por tanto, en este marco de comunicación especializada en el que se ubica el TFG, se transmite un conocimiento especializado por parte del estudiante (en su rol ahora de especialista) en una situación formal. Y, en este contexto, el trabajo académico se materializa en varios productos textuales que serán evaluados por un tribunal y que se corresponden con tres géneros textuales concretos que se transmiten por canales de comunicación distintos (escrito u oral): el trabajo académico escrito, la presentación oral para la defensa del trabajo y la presentación con diapositivas que apoya la presentación oral de la defensa. En el presente trabajo nos centramos en el primero de ellos, y en concreto en el trabajo de investigación, desde el punto de vista de la lingüística aplicada, siguiendo el enfoque indicado por Cabré (2016: 12):

La lingüística aplicada a la redacción de textos especializados es una de las ramas del estudio de la lengua que enseña cómo estructurar y describir un proyecto. Esta descripción, aplicada al proceso investigador, constituye un género textual denominado proyecto de investigación, que se encuadra en el ámbito de los géneros académicos.

En los últimos años y dado el contexto expuesto hasta aquí, diversos autores se han centrado en ofrecer recursos bibliográficos que puedan ayudar a estudiantes universitarios a redactar sus textos académicos en español, tal como indica Sánchez-Jiménez (2018: 304): 


\begin{abstract}
Por otro lado, se ha incrementado sustancialmente la cantidad de títulos publicados en este tiempo dirigidos al estudiante universitario con el objetivo último de que este mejore su escritura académica. Estos han surgido con el propósito de asistirle no solo en la redacción de los textos, pero también en la monitorización de las primeras fases de composición, desde la concepción inicial del escrito, la estructuración y la textualización, hasta el proceso de revisión intermitente que sirve para encolar todas esas partes interrelacionadas.
\end{abstract}

Estos recursos pueden estar relacionados con el proceso de escritura de textos académicos, de tesis doctorales y trabajos de investigación, y del TFG en particular, como se verá con detalle en el apartado 2.1. Sin embargo, son pocos los esfuerzos que se han realizado desde la comunidad académica por desarrollar herramientas y recursos tecnológicos que puedan servir de ayuda para la redacción de este tipo de trabajos. Los estudiantes universitarios de hoy en día son nativos digitales y, por tanto, el uso de nuevas tecnologías en el contexto académico les estimula y motiva en su proceso de aprendizaje. De hecho, la metodología de enseñanza tradicional de lengua de los libros de texto clásicos ya no se emplea como único recurso en el aula universitaria, sino que se suele combinar con herramientas y materiales electrónicos que aporten un mayor dinamismo y variedad al proceso de aprendizaje (Conroy 2010). En esta línea, existen actualmente algunas herramientas tecnológicas, basadas en el Procesamiento del Lenguaje Natural (PLN), que ayudan a corregir, revisar y/o redactar textos en diferentes lenguas, como se verá en el apartado 2.2. Estas herramientas pueden ser utilizadas con un fin didáctico, principalmente en contextos universitarios. A través de su uso, los estudiantes pueden aprender a redactar de manera interactiva, mientras escriben y revisan sus textos. Así, pueden incorporar la información lingüística que estas herramientas ofrecen en forma de sugerencias para mejorar sus escritos en diferentes niveles de la lengua (ortográfico, léxico, gramatical o pragmático, entre otros), en función de la herramienta utilizada.

En este contexto, el objetivo de este trabajo es desarrollar una herramienta TIC (Tecnologías de la Información y la Comunicación) específica para ayudar a los estudiantes en la redacción del TFG en español, en la modalidad de proyecto de investigación. La herramienta tiene forma de editor de textos en línea, y puede utilizarse gratuitamente y sin necesidad de registro. Cuenta con tres módulos, que se explican con detalle en el apartado 3.3. El Módulo 1 (Estructura y contenidos del texto) permite al estudiante insertar los apartados prototípicos del TFG, añadir títulos y contenidos habitualmente presentes en cada apartado, e incorporar fraseología relacionada con los contenidos. El Módulo 2 (Corrección ortográfica y formato) incorpora una barra superior que incluye un corrector ortográfico, así como diferentes opciones de formato mediante las cuales el estudiante tendrá acceso a las funcionalidades más habituales de los editores de texto. El Módulo 3 (Sugerencias sobre léxico y discurso) permite al estudiante procesar lingüísticamente su texto, y visualizar recomendaciones sobre aspectos lingüísticos que se podrían mejorar en su TFG, como cuestiones relacionadas con oraciones largas, siglas, unidades subjetivas, conectores discursivos, definiciones y rasgos morfosintácticos. La herramienta integra diferentes herramientas y recursos de PLN, que se detallan en el apartado 3.4.

En el apartado 2 se presenta un estado de la cuestión, por un lado, sobre la bibliografía relacionada con la redacción de textos académicos y del TFG en particular, y, por otro, sobre herramientas automáticas de ayuda a la redacción. En el apartado 3 se detalla la metodología del presente trabajo, haciendo hincapié en el marco teórico, en la selección de 
rasgos lingüísticos incorporados en la herramienta, en sus módulos y funcionalidades, y en los recursos empleados para su implementación informática. En el apartado 4 se exponen los resultados y la evaluación realizada. En el apartado 5 se incluyen las conclusiones y las líneas de trabajo futuro.

\section{ESTADO DE LA CUESTIÓN}

\subsection{La elaboración del TFG}

Existen diferentes recursos bibliográficos que pueden ser útiles para la redacción de textos académicos y profesionales en general, como el excelente manual de escritura académica y profesional de Montolío (2014), o los clásicos libros de ayuda a la redacción de Cassany (2005, 2006, 2007). Otros trabajos relevantes que se han centrado en diferentes aspectos relacionados con la escritura académica y profesional son los de Alcaraz et alii (2006), en donde se realiza un análisis de las lenguas profesionales y académicas desde diferentes enfoques (léxico, sintáctico, estilístico y discursivo, entre otros), y los de Parodi (2008, 2010, 2015), quien investiga sobre el aprendizaje del proceso de escritura académica y profesional a través del análisis exhaustivo de los diferentes géneros discursivos producidos en ambos contextos.

También ha habido autores que se han centrado específicamente en la escritura académica. Por ejemplo, en Castelló (2007a) se trata el tema de la escritura y la comunicación tanto en textos científicos como académicos. Dentro de este libro, Castelló (2007b) se centra en el proceso de composición de textos académicos. También en Núñez (2015) se pretende familiarizar al estudiante, de grado principalmente, con aspectos fundamentales de la escritura académica y contribuir al desarrollo de su competencia comunicativa y, en concreto, de las destrezas de expresión escrita. La obra más reciente sobre este tema es la de JiménezYáñez (2020), que se centra en las habilidades de comunicación académica y profesional en el ámbito universitario, y que combina explicaciones teóricas con ejercicios para poner en práctica los diferentes contenidos aprendidos por los estudiantes.

En el marco de la investigación sobre escritura académica, también se han publicado obras específicas sobre la redacción del género textual tesis doctoral o trabajo de investigación. El pionero en el campo es Eco (1997), quien publica el primer libro en español centrado en las técnicas y procedimientos de investigación, estudio y escritura para la elaboración de tesis doctorales. Posteriormente, se publica la guía de Walker (2001), que trata varias cuestiones relacionadas con la redacción de trabajos de investigación en general (como la selección del tema del trabajo o las fuentes bibliográficas, principalmente), y el breve manual de Icart et alii (2001), donde se presentan recomendaciones específicas para la elaboración y presentación de proyectos de tesis. En 2002, se publican los libros de Rigo et alii (2002), quienes ofrecen información sobre las normas formales que suelen seguirse en tesis y trabajos de investigación en general, y de Botta (2002), quien detalla normas, y técnicas de investigación y de redacción de tesis, monografías e informes. En 2003, Sabino (2003) publica una guía práctica que incluye recomendaciones y consejos de diversa índole dirigidos a estudiantes que quieran iniciarse en el mundo de la investigación, y Tolchinsky et alii (2003) publican un libro centrado en las diferentes etapas de elaboración de tesis y tesinas. Unos años después, en el manual coordinado por Pantoja (2009), se integran di- 
versos capítulos que abordan de manera muy detallada algunos temas concretos relevantes sobre la realización de tesinas, tesis y trabajos de investigación (como documentación y búsqueda de información, aspectos formales, análisis de datos cuantitativos, elaboración de cuestionarios, etc.).

Además de libros centrados en la redacción de tesis doctorales, hay otros medios que también tratan este tema, como sería el caso, por ejemplo, del blog especializado en documentación y redacción de Codina (en línea). Este blog cuenta con una sección específica sobre tesis doctorales, en donde se tratan de manera exhaustiva y a la vez muy accesible cuestiones como la relación entre la introducción y las conclusiones, la metodología de análisis cualitativo y la revisión bibliográfica.

Otros autores se han centrado en aspectos específicos de la redacción de este género textual, como puede ser su variación retórico-funcional (Martínez 2012), las formas de comunicación del conocimiento del autor en el texto (Meza 2013), las marcas estilísticas propias del autor (Savio 2010) o las relaciones discursivas que contiene (Meza y da Cunha 2019). También hay autores que analizan este género ciñéndose a un ámbito especializado, como, por ejemplo el derecho (López Escarcena 2011), el trabajo social (Tapia y Burdiles 2012), las humanidades (Tortosa 2014), la historia y la física (Martínez 2015), o la lingüística (Meza 2015), entre muchos otros.

En cuanto a la elaboración del TFG en el marco universitario español, se han publicado diferentes propuestas que analizan y describen este género textual en concreto, las cuales se detallan a continuación por orden cronológico. En primer lugar, la guía práctica para la realización este tipo de trabajos de la Universidad de Murcia, coordinada por García et alii (2012), incluye muchos aspectos que pueden extrapolarse a trabajos académicos de otras universidades españolas. En esta guía se ofrecen estrategias para la búsqueda y gestión de la información; se detallan los distintos métodos de investigación, y las técnicas de recogida y análisis de datos; se contraponen diferentes tipos de trabajos (documentales, de intervención social, empíricos y tecnológicos), y finalmente se aporta información sobre la exposición oral, la defensa y la evaluación del TFG. En segundo lugar, el libro coordinado por Ferrer et alii (2013) aborda el diseño, el desarrollo, la evaluación, la difusión y la transferencia del TFG para todas las titulaciones, y presenta una revisión del panorama internacional en el que se identifican las mejores prácticas internacionales del TFG detectadas en distintas universidades. En tercer lugar, la guía de González García et alii (2014) incluye orientaciones prácticas para los estudiantes universitarios que preparan su TFG, con el objetivo de que puedan adquirir, desarrollar y perfeccionar todas las competencias y destrezas necesarias para elaborarlo. Para ello se abordan diferentes cuestiones, como, por ejemplo, cómo elegir el tema, cómo estructurar y escribir el texto, cómo referenciar las fuentes, cómo relacionarse con el director de TFG, cómo defender el trabajo ante el tribunal y qué recursos informáticos utilizar en el trabajo. En cuarto lugar, en Barrios et alii (2016) se presenta un manual sobre la teoría y la práctica del TFG, en donde se integran tanto aportaciones sobre el tema de diferentes profesores universitarios (en relación con la gestión de recursos bibliográficos o las estrategias para las exposiciones orales, entre muchas otras) como testimonios de estudiantes que obtuvieron calificaciones excelentes en sus TFG, para ayudar a comprender debilidades comunes a la hora de elaborarlo. En quinto lugar, en el libro de da Cunha (2016) se ubica el TFG como género textual en el contexto de la comunicación especializada, se detallan las diferentes etapas incluidas en el proceso de elaboración del trabajo, se ofrecen 
pautas para su escritura (especialmente relacionadas con su estructura textual, fraseología, rasgos lingüísticos y elementos formales prototípicos), se aborda el tema de la defensa oral y se trata la posibilidad de adaptar el trabajo para publicarlo en formatos alternativos, como un artículo para una revista especializada o una comunicación en un congreso científico. En sexto lugar, en el libro coordinado por Amat et alii (2017) se incluyen múltiples capítulos, cada uno de ellos redactado por uno o varios expertos, sobre diferentes aspectos relacionados con la elaboración de trabajos investigación de diferentes niveles, entre ellos el TFG. Algunos de los aspectos abordados son la selección del director del trabajo, la revisión de la literatura, la formulación de la pregunta de investigación, la elaboración del marco teórico o el diseño de la investigación, entre otros. Finalmente, en Cierlica (2018) se ofrece un libro electrónico con un estilo ameno y muy desenfadado, de carácter eminentemente práctico, que buscar explicar las diferentes etapas del TFG, desde la elección del tema hasta la redacción y revisión del manuscrito, pasando por la búsqueda de materiales y selección de bibliografía.

También algunas universidades o estudios específicos han publicado en línea recursos o guías para ayudar a sus estudiantes a elaborar trabajos académicos. Es el caso, por ejemplo, de la guía redactada por Anduiza y Méndez (en línea) en el área de Ciencia Política y de la Administración de la Universidad de Murcia; de las sugerencias para la realización de trabajos académicos y científicos propuestas por Sánchez Herrera (en línea) del Departamento de Sociología de la Universidad de La Laguna; o de la guía de buenas prácticas para la elaboración de los TFG de los diferentes grados de la Facultad de Filosofía y Letras de la Universidad de Cádiz, coordinada por Rodríguez-Piñeiro (2014). En el caso de la Universidad Nacional de Educación a Distancia (UNED), dada su metodología en línea, se ha optado por habilitar un espacio en la Biblioteca virtual ${ }^{1}$ donde se puede acceder a diversos vídeos en que diferentes profesores tratan temas relacionados con la elaboración del TFG, como por ejemplo la búsqueda de información, los aspectos formales o la citación de referencias bibliográficas.

Como apunte final, es de destacar que en el ámbito latinoamericano está creciendo el interés por la investigación sobre la redacción del TFG (también llamado, entre otras formas, tesis de grado) en sus universidades. Un ejemplo representativo es el de Chile, donde recientemente se ha publicado una guía muy completa sobre la redacción del TFG partiendo de la escritura desde la pedagogía del género (Venegas et alii 2015).

Todas las obras mencionadas en este apartado son válidas e interesantes y, además, son complementarias, ya que cada una trata aspectos concretos que pueden resultar de utilidad a quien desee iniciarse en el mundo de la investigación o elaborar un TFG. En el marco del presente trabajo y de cara al desarrollo de una herramienta TIC para la redacción del TFG en español, nos basamos en da Cunha (2016), ya que es una de las obras más recientes cuyo contenido se ajusta a los criterios del Plan Bolonia en el contexto universitario español. Asimismo, el libro se centra específicamente en la estructura del TFG, y ofrece una tabla muy completa que detalla los apartados prototípicos de la estructura de este género textual, junto con la especificación de los contenidos que se incluyen habitualmente en cada uno de los apartados, lo cual es una información fundamental para su integración en la herramienta

1 Disponible desde: http://portal.uned.es/portal/page?_pageid=93,55541853\&_dad=portal\&_schema=PORTAL (01-05-2020). 
TIC desarrollada en este trabajo, como se verá en el apartado 3.3. Además, el libro aporta información sobre la fraseología empleada en este género textual y sobre aspectos relacionados con una redacción adecuada, que también se integrarán en la herramienta. Se trata de uno de los libros sobre el TFG más recomendado por las diferentes universidades españolas en distintos grados, lo cual es un criterio de fiabilidad adicional para su selección.

\subsection{Herramientas automáticas de ayuda a la redacción}

En el apartado 2.1 se ha visto que en los últimos años se han publicado libros, guías y manuales sobre el proceso de escritura en español de textos académicos en general y del TFG en particular. Estos recursos con toda seguridad serán útiles para los estudiantes universitarios que se enfrenten a la elaboración de este género textual. No obstante, como ya se avanzaba en el apartado 1, el desarrollo de herramientas y recursos tecnológicos que puedan servir como complemento a la hora estructurar, redactar y revisar un TFG es un tema poco explorado, especialmente en español.

En inglés, lengua para la que se desarrollan la mayor parte de los recursos tecnológicos hoy en día, tenemos algunos ejemplos de herramientas de redacción asistida que ayudan a escribir textos científicos y académicos, como por ejemplo $\mathrm{SWAN}^{2}, \mathrm{CALeSE}^{3}$ y eWriting $\mathrm{Pal}^{4}$. También se han desarrollado diversos sistemas que ayudan a corregir errores grama-

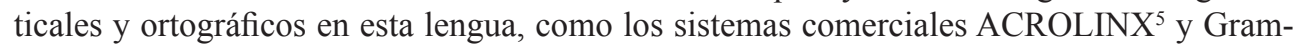
marChecker ${ }^{6}$, entre otros. Asimismo, existen sistemas comerciales que permiten corregir errores en textos generales en inglés y que tienen en cuenta las características de ciertos géneros textuales, como es el caso de Grammarly.

En el caso del español, también existen sistemas que detectan en textos generales errores gramaticales, ortográficos e incluso algunos de estilo, como es el sistema comercial Stilus ${ }^{8}$ y el sistema de código abierto para múltiples lenguas LanguageTool ${ }^{9}$. Para la ayuda en la redacción de textos académicos universitarios en español, se encuentra la herramienta Estilector $^{10}$, que permite revisar lingüísticamente los textos escritos por los estudiantes. Concretamente, la herramienta ofrece un informe sobre la cantidad de palabras, caracteres y párrafos del texto, proporciona una clasificación de los nombres propios detectados, y contabiliza y clasifica los conectores discursivos encontrados. Asimismo, analiza cada párrafo del texto, y detecta palabras repetidas, unidades léxicas demasiado generales (ej. hacer) y algunos problemas en la puntuación, entre otras cuestiones.

Otro caso interesante es el de Linguakit ${ }^{11}$, un corrector que analiza el texto buscando errores ortográficos, léxicos, gramaticales o de estilo. Actualmente, únicamente funciona para el gallego, pero, según los autores, su arquitectura se podría adaptar a otras lenguas,

\footnotetext{
2 https://cs.joensuu.fi/swan/ (01-05-2020).

3 http://www.nilc.icmc.usp.br/calese/ (01-05-2020).

$4 \mathrm{http}: / /$ www.ewritingpal.com/ (01-05-2020).

5 http://www.acrolinx.com/ (01-05-2020).

6 https://www.e-uned.es/correctme/cm_english/ (01-05-2020).

7 http://www.grammarly.com/ (01-05-2020).

8 http://www.mystilus.com/ (01-05-2020).

9 https://www.languagetool.org/ (01-05-2020).

$10 \mathrm{http} / /$ www.estilector.com/ (01-05-2020).

$11 \mathrm{https} / / /$ linguakit.com/es/supercorrector (01-05-2020).
} 
como el español, en caso de tener los recursos necesarios, como diccionarios y analizadores morfosintácticos (Gamallo et alii 2015).

No obstante, por un lado, ninguno de los sistemas mencionados, ni siquiera los desarrollados para el inglés, se centra en la elaboración del género textual TFG. Ninguno de ellos permite estructurar el documento, asignando títulos, contenidos y fraseología habituales en este género textual a sus diferentes apartados prototípicos. Por otro lado, en el caso de los sistemas mencionados para el español, la revisión lingüística realizada por Stilus y LanguageTool no incluye recomendaciones avanzadas con información léxica y discursiva, al tratarse de correctores principalmente ortográficos y gramaticales. En el caso de Estilector, sí se ofrecen algunas funciones interesantes en este sentido. Por ejemplo, como se ha mencionado, esta herramienta detecta los conectores discursivos empleados en el texto y ofrece un informe donde los clasifica según el tipo de relación discursiva que expresan. Sin embargo, no ofrece sugerencias de mejora sobre este tema, más allá de la consulta de bibliografía recomendada.

\section{METODOLOGÍA}

\subsection{Marco teórico}

En la fase de conceptualización de la herramienta TIC, se decidió incorporar información correspondiente a tres niveles lingüísticos (textual, léxico y discursivo), que ayudasen al estudiante tanto a estructurar y a redactar su TFG, como a revisar aspectos léxicos y discursivos que se podrían mejorar en el texto. Para abordar estos niveles se emplearon tres marcos teóricos complementarios, que se detallan a continuación.

En primer lugar, con respecto al nivel textual, se partió de los trabajos de van Dijk (1977, 1989), quien afirma que los géneros textuales siguen un patrón claramente codificado y ampliamente aceptado. Por ejemplo, un artículo de investigación suele incluir ciertos apartados prototípicos: Introducción, Estado de la cuestión, Metodología, Resultados y Conclusiones. Este autor, además, define la superestructura como la estructura organizativa textual, que varía dependiendo del tipo de texto y que, en general, se muestra mediante diferentes apartados, que suelen incluir títulos y diferentes contenidos prototípicos. Estos contenidos vendrían a ser similares al concepto moves (movidas) de Swales (1990), es decir, fragmentos de texto que sirven para una función comunicativa y semántica particular.

En segundo lugar, en cuanto al nivel léxico, se siguió la Teoría Comunicativa de la Terminología (TCT) de Cabré (1999), que es una teoría de la terminología y del discurso especializado que pone de relieve la dimensión comunicativa de los textos de especialidad. Según la TCT, los géneros textuales producidos en ámbitos especializados presentan algunas características globales, como la precisión, la concisión, la sistematicidad, la impersonalidad y la objetividad. Estas características se hacen evidentes en los textos por medio de diferentes rasgos lingüísticos. Por ejemplo, la concisión se puede lograr a través del uso de siglas y la objetividad se puede alcanzar evitando marcadores de subjetividad, entre otras estrategias.

En tercer lugar, en relación con el nivel discursivo, se empleó la Rhetorical Structure Theory (RST) de Mann y Thompson (1988), que es una teoría de organización textual que permite describir un documento caracterizando su estructura mediante las relaciones retóricas que mantienen sus segmentos discursivos. Estas relaciones pueden ser núcleo- 
satélite o multinucleares. En las relaciones núcleo-satélite hay dos elementos: uno de ellos es más relevante de cara a los propósitos del emisor (el núcleo), mientras que el otro (el satélite) aporta una información adicional sobre el núcleo. En las relaciones multinucleares, en cambio, puede haber más de dos elementos, todos ellos núcleos, que se relacionan al mismo nivel, es decir, todos tienen la misma importancia de cara a los propósitos del autor. Estos elementos se llaman segmentos discursivos o elementary discourse units (EDUs), que pueden definirse como indican Tofiloski et alii (2009: 77): "Discourse segmentation is the process of decomposing discourse into elementary discourse units (EDUs), which may be simple sentences or clauses in a complex sentence, and from which discourse trees are constructed". Por ejemplo, los ejemplos (1) y (2) incluyen cada uno una oración extraída de un TFG del ámbito de la lingüística ${ }^{12}$ que se podría dividir en dos segmentos discursivos (indicados entre corchetes, junto con el número de segmento):

(1) [Además, en lo que respecta a las instrucciones, el material de una de las editoriales incluye actividades que se tienen que desarrollar de manera externa a la plataforma online,] SEGMENTO1 [pero esto no se indica en ninguna parte de las instrucciones.]SEGMENTO2

(2) [Las cinco editoriales proponen el trabajo de un contenido coherente con los objetivos del currículum y las destrezas que se espera que desarrollen los alumnos,]SEGMENTo1 [es decir, el material está, en todos los casos, adecuado al nivel de conocimiento de los alumnos.] SEGMENTO2

En la propuesta original de la RST se incluyen 25 relaciones discursivas. ${ }^{13}$ En el presente trabajo se seleccionaron ocho de ellas para el análisis: siete relaciones núcleo-satélite (antítesis, causa, concesión, condición, propósito, reformulación y resumen) y una relación multinuclear (contraste). Esta selección se realizó a través de la búsqueda de relaciones discursivas que aparecen de manera frecuente en el corpus de referencia anotado con relaciones discursivas en español, el RST Spanish Treebank ${ }^{14}$, y que a la vez se evidencian habitualmente por medio de conectores discursivos, tal como se recoge en da Cunha et alii (2012a). Precisamente los conectores discursivos tienen una gran relevancia en el marco de la RST, pues, en función de la lengua, suele haber un porcentaje de relaciones discursivas que se expresan de manera explícita a través de estas unidades y un porcentaje que lo hace de manera implícita (es decir, sin el uso de conectores). Investigaciones previas indican que entre el $20 \%$ y el $43 \%$ de las relaciones discursivas están marcadas a través de conectores en español, inglés y euskera, en función del corpus empleado (Taboada 2006; Iruskieta y da Cunha 2010). En el marco de nuestro trabajo y de cara a integrarlos en la herramienta, hacemos una diferencia entre conectores discursivos intraoracionales (es decir, conectores que enlazan segmentos dentro de una misma oración) y conectores interoracionales (es decir, conectores que enlazan diferentes oraciones). Por ejemplo, la oración mostrada en el ejemplo (1) incluye un conector intraoracional que encabeza el segmento 2, "pero", que

12 Escrig, A. (2019). Análisis de la integración del material TIC/TAC en la asignatura de Lengua Castellana y Literatura. Trabajo de Fin de Grado (TFG). Grado en Lenguas Aplicadas, Universitat Pompeu Fabra, Barcelona. Accesible desde e-Repositori: https://repositori.upf.edu/ (01-05-2020).

13 Para más información sobre estas relaciones y su definición, se recomienda acceder a la web oficial de la RST: http://www.sfu.ca/rst/index.html (01-05-2020).

14 Accesible desde: http://www.corpus.unam.mx/rst/index_es.html (01-05-2020). 
marca una relación de antítesis; por su parte, la oración del ejemplo (2) incluye también un conector intraoracional, en este caso "es decir", que refleja una relación de reformulación. Si estas oraciones se dividiesen en dos oraciones diferentes, más breves, esos dos conectores intraoracionales deberían sustituirse por conectores interoracionales que expresasen la misma relación discursiva, como por ejemplo "sin embargo" y "en otras palabras", respectivamente, tal como se recoge en los ejemplos (3) y (4), donde ambos conectores se han marcado en cursiva:

(3) [Además, en lo que respecta a las instrucciones, el material de una de las editoriales incluye actividades que se tienen que desarrollar de manera externa a la plataforma online.] SEGMENTO1 [Sin embargo, esto no se indica en ninguna parte de las instrucciones.]SEGMENTO2

(4) [Las cinco editoriales proponen el trabajo de un contenido coherente con los objetivos del currículum y las destrezas que se espera que desarrollen los alumnos.]SEGMENTO1 [En otras palabras, el material está, en todos los casos, adecuado al nivel de conocimiento de los alumnos.]SEGMENTO2

\subsection{Selección de rasgos lingüísticos para incorporar en la herramienta TIC}

Como ya se avanzó en el apartado 1, la herramienta TIC incorpora 3 módulos. Para el diseño del Módulo 1 (Estructura y contenidos del texto), se tienen en cuenta los conceptos introducidos por van Dijk $(1977,1989)$ y Swales (1990) mencionados en el apartado 3.1. Este módulo permite al estudiante insertar los apartados prototípicos del TFG, añadir títulos y contenidos habitualmente presentes en cada apartado, e incorporar fraseología relacionada con los contenidos. Para la selección de estos elementos concretos en el género textual TFG, nos basamos en el trabajo de da Cunha (2016). Específicamente, para determinar los apartados y contenidos prototípicos utilizamos como referencia la Tabla 1.

\begin{tabular}{|c|c|}
\hline Apartados prototípicos & Contenidos prototípicos \\
\hline Portada & $\begin{array}{ll}\checkmark & \text { Título } \\
\checkmark & \text { Datos académicos: nombre y logo de la institución, nombre del } \\
\text { programa académico, tipo de trabajo académico (de grado, de } \\
\text { máster, etc.), nombre y apellidos del autor, nombre y apellidos del } \\
\text { director (y, en su caso, del codirector), ciudad, fecha, etc. }\end{array}$ \\
\hline $\begin{array}{l}\text { Agradecimientos } \\
\text { (opcional) }\end{array}$ & $\begin{array}{l}\checkmark \quad \text { Reconocimiento al apoyo de la institución académica, grupo de } \\
\text { investigación, programa de estudios, becas recibidas, etc. } \\
\checkmark \quad \text { Reconocimiento al apoyo de personas concretas, tanto del ámbito } \\
\text { académico como del familiar o personal }\end{array}$ \\
\hline Índice & $\begin{array}{l}\checkmark \text { Títulos de cada apartado numerados, con indicación de la página } \\
\text { donde comienza cada uno }\end{array}$ \\
\hline Resumen + abstract & $\begin{array}{l}\checkmark \text { Resumen en la lengua del trabajo que incluye los contenidos } \\
\text { principales del trabajo } \\
\checkmark \quad \text { Abstract (resumen traducido al inglés) }\end{array}$ \\
\hline $\begin{array}{l}\text { Palabras clave }+ \\
\text { keywords }\end{array}$ & $\begin{array}{l}\quad \text { Términos en la lengua del trabajo que mejor representan el tema del } \\
\text { mismo } \\
\checkmark \quad \text { Keywords (palabras clave equivalentes en inglés) }\end{array}$ \\
\hline
\end{tabular}




\begin{tabular}{|l|ll|}
\hline Introducción & $\checkmark$ & Contexto del trabajo \\
& $\checkmark$ Motivación \\
$\checkmark$ & Objetivos \\
$\checkmark$ & Hipótesis o ideas de partida \\
$\checkmark$ & Aplicaciones posibles \\
& $\checkmark$ Estructura del trabajo
\end{tabular}

Tabla 1. Apartados y contenidos prototípicos de la estructura de un TFG (da Cunha 2016: 75-76)

En cuanto a la fraseología típicamente empleada para referirse a los diferentes contenidos de la Tabla 1, nos basamos en la misma obra. Por ejemplo, en el apartado Introducción, para introducir el contenido Objetivos, se ofrecen diversas propuestas, como por ejemplo:

- El objetivo general de este TFG es

- El objetivo principal de este TFG es

- Este TFG persigue los objetivos que se indican a continuación:

- Este TFG recoge los siguientes objetivos:

- En este TFG se incluyen los objetivos siguientes:

- Los objetivos generales de este TFG son:

- Los objetivos específicos de este TFG son: 
Para el diseño del Módulo 2 (Corrección ortográfica y formato) y del Módulo 3 (Sugerencias sobre léxico y discurso) se tienen en cuenta los marcos teóricos de la TCT y de la RST, en relación con los rasgos léxicos y discursivos, respectivamente, como se mencionó en el apartado 3.1. El Módulo 2 permite al estudiante revisar ortográficamente su TFG, tarea indispensable a la hora de elaborar este tipo de trabajos, ya que la adecuación ortográfica y gramatical del documento se considera un requisito básico en este género textual, que normalmente se tiene en cuenta para la evaluación. El Módulo 3 ofrece recomendaciones sobre aspectos lingüísticos que se podrían mejorar en el TFG del estudiante. Para la selección de las recomendaciones que se implementarán en la herramienta TIC, tenemos en cuenta las palabras de Cabré (2016: 13) cuando se refiere a las características fundamentales de un TFG, como son la adecuación gramatical y estilística, la precisión, la concisión, la sistematicidad y la objetividad:

El proyecto debe ser, asimismo, gramaticalmente adecuado, en el sentido de usar la lengua de redacción de manera correcta y presentar la terminología más habitual sobre el tema y el proceso de investigación. Finalmente, como todo texto especializado, el texto del proyecto debe ser también estilísticamente adecuado, de manera que la redacción respete factores como la precisión, la concisión y la sistematicidad, al lado de la objetividad y la máxima distancia subjetiva en relación con los datos.

Así, se seleccionaron rasgos lingüísticos que ayudan a potenciar estas características fundamentales del TFG y, además, cuya implementación informática fuese factible con recursos de PLN para el español. Por ejemplo:

- Para potenciar la concisión, la herramienta TIC detecta en el TFG oraciones muy largas y ofrece propuestas de segmentación discursiva, con el objetivo de que el estudiante decida si quiere segmentarlas en otras oraciones más breves.

- Para potenciar la precisión, detecta las siglas que no se acompañan de su correspondiente término desplegado la primera vez que aparecen en el texto, con el objetivo de que el estudiante corrija esta cuestión.

- Para potenciar la objetividad, detecta en el texto unidades léxicas indicadoras de subjetividad, con el objetivo de que el estudiante decida si quiere eliminarlas.

- Para potenciar la sistematicidad, detecta los verbos en $1^{\text {a }}$ persona del singular y del plural, con el objetivo de que el estudiante revise si su uso es sistemático cuando se introduce él mismo como autor del trabajo.

- Para potenciar la concisión, detecta la inexistencia de definiciones en el texto, con el objetivo de que el estudiante se plantee la conveniencia de añadir alguna en su trabajo.

Todas las recomendaciones de la herramienta se detallan e ilustran en el apartado 3.3. Como se verá, también se incluyen recomendaciones que ofrecen información sobre conectores discursivos, que se han añadido principalmente porque son unidades que ayudan a cohesionar el TFG y los estudiantes en muchas ocasiones tienen carencias sobre este tema. Precisamente, tal como indican Villar et alii (2018: 610): ${ }^{15}$

15 No entramos aquí en la discusión terminológica sobre el empleo de conector discursivo o marcador discursivo, pues no es el objetivo de este trabajo. Para saber más sobre estas unidades en español recomendamos al lector los 
En el proceso de redacción es necesario el uso de los marcadores discursivos que, muchas veces, los estudiantes universitarios no emplean. [...]. Así, para estos alumnos universitarios, es muy importante que sepan utilizar los marcadores discursivos en la redacción de textos; siendo necesario plantear estrategias que mejoren este procedimiento.

En este sentido, los trabajos de Errázuriz $(2012,2014)$ demuestran que existe una correspondencia entre la adecuación de los conectores discursivos y la progresión lógica de los ensayos de los estudiantes universitarios. Precisamente, en los últimos años se ha investigado sobre el desarrollo de nuevas actividades para la enseñanza de estas unidades, por ejemplo en el contexto de español como lengua extranjera (Corral 2010).

\subsection{Módulos y funcionalidades de la herramienta TIC}

La herramienta TIC desarrollada tiene forma de editor de textos en línea, y puede utilizarse gratuitamente y sin necesidad de registro. ${ }^{16}$ Cuenta con un manual de uso donde se detallan y ejemplifican sus diferentes funcionalidades. ${ }^{17}$ Una vez el estudiante entra en el editor en línea (véase Figura 1), puede comenzar a redactar su TFG en la hoja de texto central, utilizando los tres módulos de ayuda con los que cuenta la herramienta, que se detallan en este apartado.

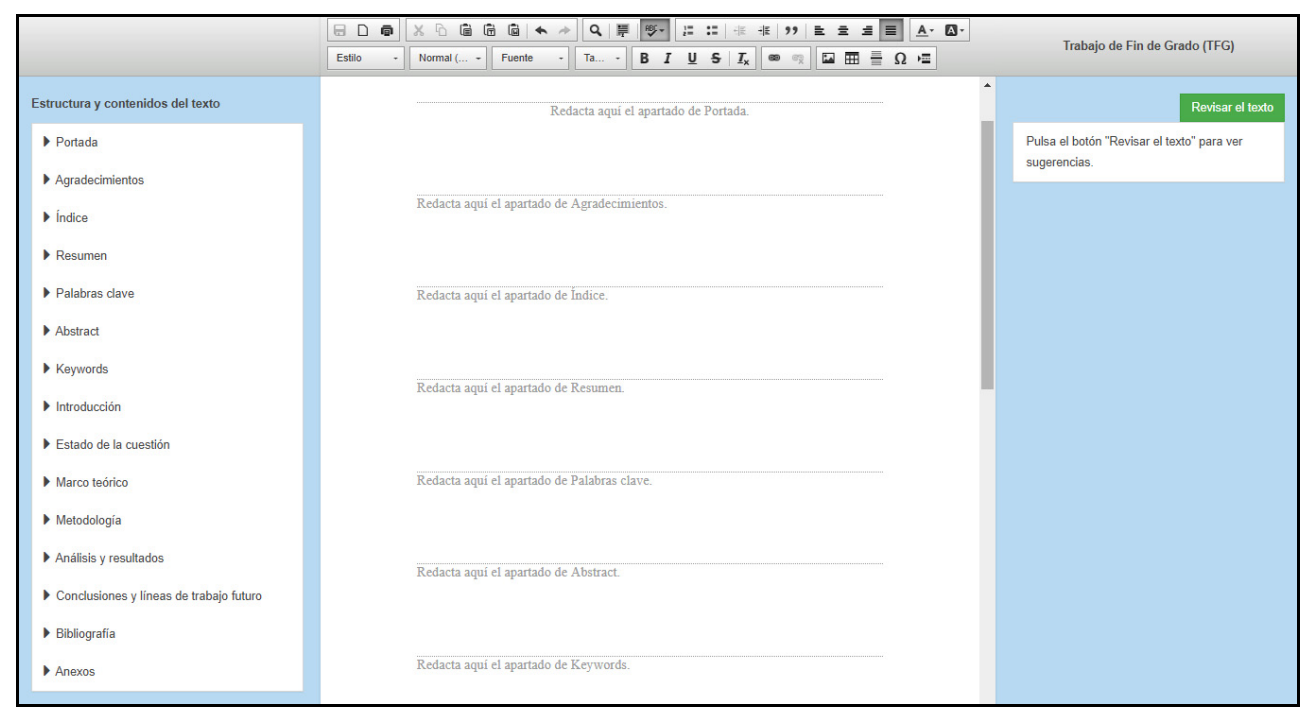

Figura 1. Captura de la pantalla inicial del editor de textos en línea de la herramienta

trabajos de referencia siguientes: Portolés (1998), Montolío (2001), Santos Río (2003), Cuenca (2006), y Briz et alii (2008).

16 Accesible desde: http://sistema-artext.com/ (01-05-2020).

17 Accesible desde: http://sistema-artext.com/doc/manual.pdf (01-05-2020). 
La herramienta incorpora un módulo que ayuda a estructurar el documento y a comenzar a redactarlo. Este módulo se encuentra en la columna izquierda de la pantalla y permite:

- Insertar los apartados prototípicos del documento.

- Añadir títulos y contenidos habitualmente presentes en cada apartado.

- Incorporar fraseología relacionada con los contenidos.

Tal como muestra la Figura 1, lo primero que visualiza el estudiante en la columna izquierda es la lista de apartados sugeridos para un TFG. Cada apartado está precedido por el símbolo $\downarrow$. Al hacer clic sobre cada uno de los apartados, el símbolo que los precede cambia de dirección $(\nabla)$ y automáticamente se despliega debajo de cada uno de ellos una lista que incluye los contenidos que se recomienda insertar en cada apartado, en el orden propuesto. Estos contenidos también incluyen el título del apartado. En la Figura 2 se ofrece un ejemplo, en donde se muestran los diferentes contenidos del apartado Introducción.

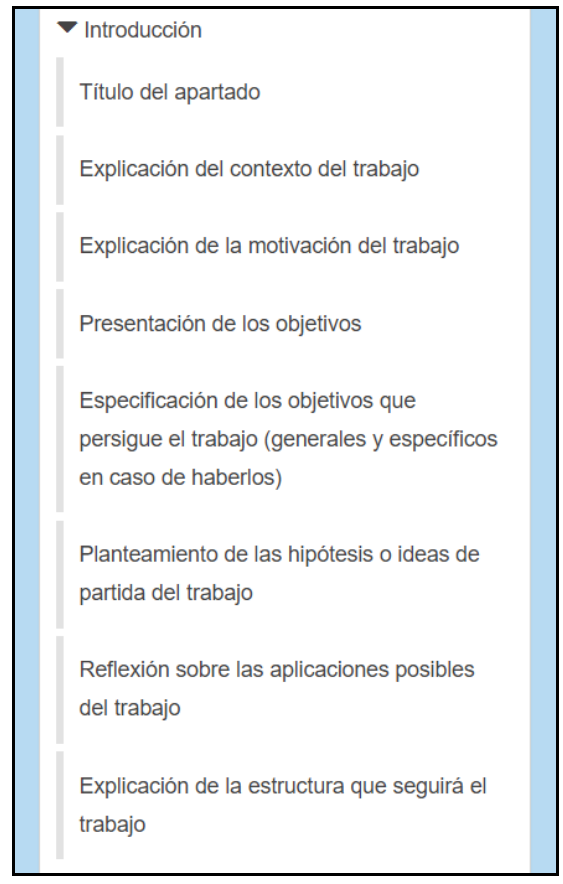

Figura 2. Detalle de una captura de pantalla del Módulo 1 de la herramienta, donde se muestra el apartado Introducción y sus contenidos prototípicos

A su vez, al hacer clic sobre un contenido, se muestra una lista de frases que podrían ayudar a redactarlo, que no aparecen en un orden determinado. Por ejemplo, en el apartado 
Metodología, para el contenido Presentación de la metodología, la herramienta ofrece las siguientes opciones: ${ }^{18}$

- La metodología de este trabajo sigue las [pasos/fases/etapas] indicadas a continuación.

- La metodología seguida en este trabajo incluye [nº] [pasos/fases/etapas].

- La metodología del trabajo es la siguiente:

\section{MÓDULO DE CORRECCIÓN ORTOGRÁFICA Y FORMATO}

La herramienta incorpora una barra superior que incluye diferentes opciones de formato mediante las cuales el estudiante tendrá acceso a las funcionalidades más habituales de los editores de texto, como seleccionar el tamaño de letra y la fuente, asignar estilos, insertar tablas, hacer listas, copiar, pegar, buscar, etc. (véase Figura 1). Asimismo, en esta barra de formato se ha integrado un corrector ortográfico de código abierto (WebSpellChecker Ltd).

\section{Módulo DE SUGerenCiAS SOBRE LÉXICO Y DISCURSO}

La herramienta cuenta con un módulo de sugerencias que permite al estudiante procesar lingüísticamente su texto, y visualizar recomendaciones sobre cuestiones relacionadas con aspectos lingüísticos (léxicos y discursivos, principalmente) que se podrían mejorar. Para obtenerlas, el estudiante debe tener escrito su texto en el editor. Por ejemplo, en la Figura 3 se incluye un texto ficticio que podría ser parte de un TFG.

Desgraciadamente este tema ha sido acordado en la CEP (Cumbre Europea Permanente) este año en colaboración
con los países en vías de desarrollo. En esa Cumbre Europea Permanente se abordó la decisión tomada por unos
países determinados. En mi opinión, esta decisión internacional se tomó sin reflexionar lo suficiente, porque
evidentemente se deseaba un consenso entre todas las partes implicadas, pero ese acuerdo podría haberse obtenido
a través de una reflexión más profunda que implicase más actores en el proceso de toma de decisiones. Estoy al
tanto de esta información porque he realizado una búsqueda bibliográfica exhaustiva. En ella, hemos detectado las
decisiones tomadas en ese proceso, en que se tuvieron en cuenta especialmente a los países en vías de desarrollo
representados por la OIPVD.
En general debe tenerse en cuenta que en los países mencionados hay algunas normativas que están obsoletas
porque no se han vuelto a tratar por ningún comité, es decir, no habido especialistas en el ámbito que hayan
retomado y actualizado dichos documentos. Sin embargo, en otros países con distinto tipo de recursos e intereses
la situación es diferente. Estos países se podrían tomar como modelo. Es decir, podríamos tener en cuenta las
políticas adoptadas por otros países. No obstante, la realidad es que nos cuesta entender que nuestras decisiones
no siempre son las más acertadas
En otras comunidades, hace tiempo que negocian sobre cómo alcanzar un pacto para la regeneración de ideas en
relación con esta materia. Deberíamos fijarnos en ellos para observar maneras distintas y complementarias de
gestionar nuestros procesos de toma de decisiones al respecto, es decir, no caer en los errores de siempre.
En conclusión, sugiero que hagamos una reflexión sobre esta cuestión en profundidad. Debemos ser conscientes
de la repercusión que pueden tener nuestros actos, es decir, meditar bien nuestras decisiones. Es necesario que
logremos un consenso entre la mayor parte de países posibles, para no hacer de esta decisión un acto político y
llegar realmente a acuerdos con un consenso mayoritario.

Figura 3. Texto ficticio utilizado para ejemplificar las recomendaciones de la herramienta

18 Los corchetes indican una información variable en la frase, que el estudiante debe insertar (ej. el número de fases de la metodología) o seleccionar (ej. la unidad léxica pasos, fases o etapas). 
Una vez escrito el texto, el estudiante debe hacer clic sobre el botón Revisar el texto de la columna derecha de la pantalla. Entonces, la herramienta le ofrecerá en esa misma columna diferentes recomendaciones de mejora del texto, como se recoge en la Figura 4.

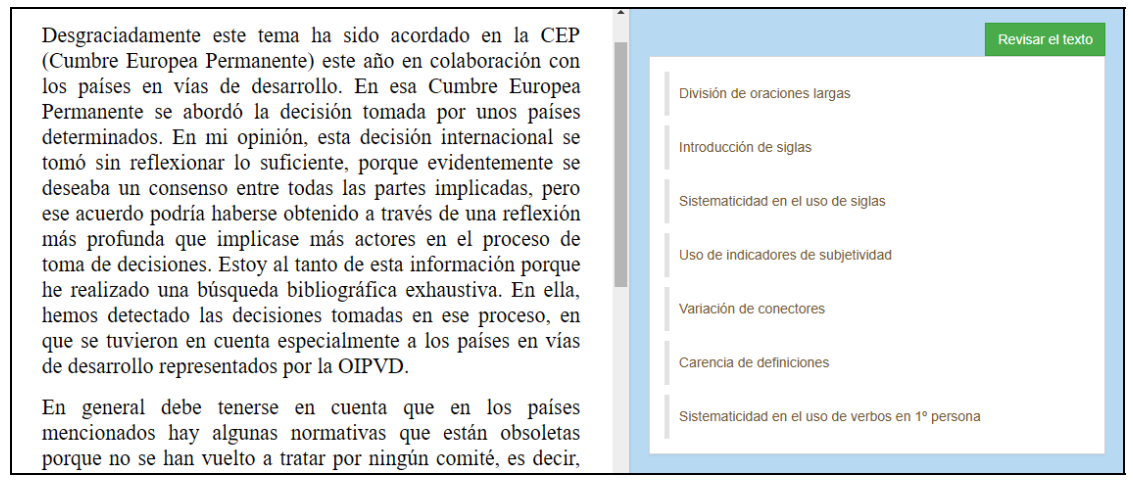

Figura 4. Detalle de una captura de pantalla de la herramienta que muestra las recomendaciones obtenidas al procesar un texto

En función de la recomendación sobre la que el estudiante haga clic, en el texto que ha escrito en pantalla aparecerán marcadas con colores diferentes cuestiones, como oraciones largas, siglas, conectores, etc. A continuación se detallan las recomendaciones de mejora ofrecidas al estudiante al procesar el texto, junto con un ejemplo representativo de cada una de ellas:

a) "División de oraciones largas. Parece que las oraciones marcadas podrían dividirse en otras más cortas. Te recomendamos que lo hagas. Haz clic en cada oración para ver dónde podrías segmentarla."

En este caso, la herramienta destaca en el texto las oraciones consideradas demasiado largas para un TFG (véase Figura 5). Para ello, se toma como referencia el umbral de 25 de palabras propuesto por Mari (1998) en el contexto de la redacción científica.

Además, como se verá en el apartado 3.4, la herramienta integra un segmentador discursivo (da Cunha et alii 2012b). En caso de que este segmentador logre dividir automáticamente la oración en segmentos discursivos más breves, la herramienta también ofrece esta información al estudiante en la columna derecha. Veamos por ejemplo la siguiente oración (extraída del texto de la Figura 3):

En mi opinión, esta decisión internacional se tomó sin reflexionar lo suficiente, porque evidentemente se deseaba un consenso entre todas las partes implicadas, pero ese acuerdo podría haberse obtenido a través de una reflexión más profunda que implicase más actores en el proceso de toma de decisiones.

En este caso, la herramienta propone dividir esta oración larga en tres oraciones más breves, teniendo en cuenta los tres segmentos siguientes (véase Figura 5): 
1. En mi opinión, esta decisión internacional se tomó sin reflexionar lo suficiente,

2. porque evidentemente se deseaba un consenso entre todas las partes implicadas,

3. pero ese acuerdo podría haberse obtenido a través de una reflexión más profunda que implicase más actores en el proceso de toma de decisiones.

Asimismo, en caso de que los segmentos propuestos comiencen por conectores discursivos intraoracionales, la herramienta propone al estudiante opciones de conectores interoracionales alternativos que expresen la misma relación discursiva. Para ello, la herramienta incluye una lista de este tipo de unidades, extraídas de da Cunha et alii (2012a). Siguiendo con la misma oración, en el texto se marcarían dos conectores: porque (que refleja una relación de causa) y pero (que refleja una relación de antítesis). Por ejemplo, si el estudiante hace clic en pero, verá en la columna de la derecha las siguientes propuestas de conectores interoracionales alternativos, que expresan la misma relación de antítesis: Con todo, De todas formas, De todas maneras, De todos modos, No obstante y Sin embargo ${ }^{19}$ (véase Figura 5). De esta manera, el estudiante puede decidir si desea dividir la oración marcada en otras oraciones más breves y mantener explícita la relación discursiva que existe entre ellas a través de un conector. Es el estudiante quien decide en todo momento qué recomendaciones desea incorporar en su texto. Para hacerlo, simplemente debe realizar los cambios directamente en la hoja del editor de texto.

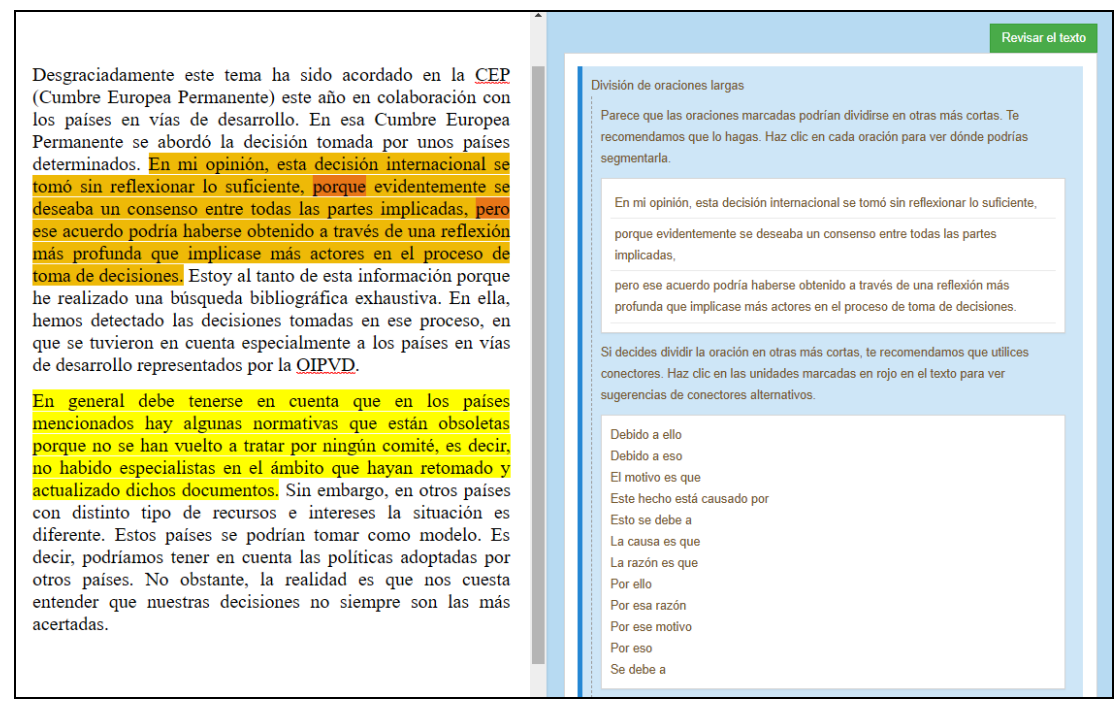

Figura 5. Detalle de una captura de pantalla de la herramienta que refleja la recomendación División de oraciones largas

b) "Introducción de siglas. Las unidades marcadas parecen siglas. Si es así, ten en cuenta que la primera vez que se utiliza una sigla en un texto suele ir acompañada del término desplegado."

19 En este caso, estos conectores aparecen con mayúscula inicial puesto que irán a principio de oración. 
En este caso, la herramienta marca en el texto las siglas que no se acompañan de su correspondiente término desplegado la primera vez que aparecen en el TFG, lo cual no es recomendable. Por el momento, la herramienta detecta únicamente siglas propias (Giraldo 2008), es decir, aquellas que están formadas únicamente por las unidades léxicas incluidas en la estructura sintagmática del término desplegado, como por ejemplo $O N U$, que se corresponde con Organización de las Naciones Unidas. En la Figura 6 puede verse que la herramienta ha detectado un caso, OIPVD.

\begin{tabular}{|c|c|}
\hline Desgraciadamente este tema ha sido acordado en la CEP & Revisar el texto \\
\hline $\begin{array}{l}\text { los países en vías de desarrollo. En esa Cumbre Europea } \\
\text { Permanente se abordó la decisión tomada por unos paises }\end{array}$ & División de oraciones largas \\
\hline $\begin{array}{l}\text { determinados. En mi opinión, esta decisión internacional se } \\
\text { tomó sin reflexionar lo suficiente, porque evidentemente se }\end{array}$ & \multirow{2}{*}{$\begin{array}{l}\text { Introducción de siglas } \\
\text { Las unidades marcadas parecen siglas. Si es asi, ten en cuenta que la primera vez que } \\
\text { se utiliza una sigla en un texto suele ir acompañada del término desplegado. }\end{array}$} \\
\hline $\begin{array}{l}\text { deseaba un consenso entre todas las partes implicadas, pero } \\
\text { ese acuerdo podría haberse obtenido a través de una reflexión }\end{array}$ & \\
\hline más profunda que implicase más actores en el proceso de & Ejemplos: \\
\hline he realizado una búsqueda bibliográfica exhaustiva. En ella. & Universidad Nacional de Educación a Distancia (UNED) \\
\hline hemos detectado las decisiones tomadas en ese proceso, en & EPOC (enfermedad pulmonar obstructiva crónica) \\
\hline de desarrollo representados por la OIPVD. & Sistematicidad en el uso de siglas \\
\hline
\end{tabular}

Figura 6. Detalle de una captura de pantalla de la herramienta que refleja la recomendación Introducción de siglas

c) "Sistematicidad en el uso de siglas. Las unidades marcadas parecen el término desplegado de siglas que utilizas en el texto. Si es así, ten en cuenta que, una vez se introduce una sigla en un texto, se suele seguir utilizando la sigla y no el término desplegado."

Esta recomendación también está relacionada con las siglas. En esta ocasión, la herramienta marca en el texto las ocurrencias de términos desplegados que ya habían sido introducidos en el TFG previamente junto con su correspondiente sigla, lo cual es desaconsejable. En la Figura 7 puede verse un ejemplo, en que la unidad Cumbre Europea Permanente está marcada dos veces. La primera vez aparece en una oración junto con su sigla entre paréntesis, correctamente. Sin embargo, también aparece marcada en la siguiente oración, ya que se quiere hacer notar al estudiante que sería más conveniente sustituir esta unidad por su sigla.

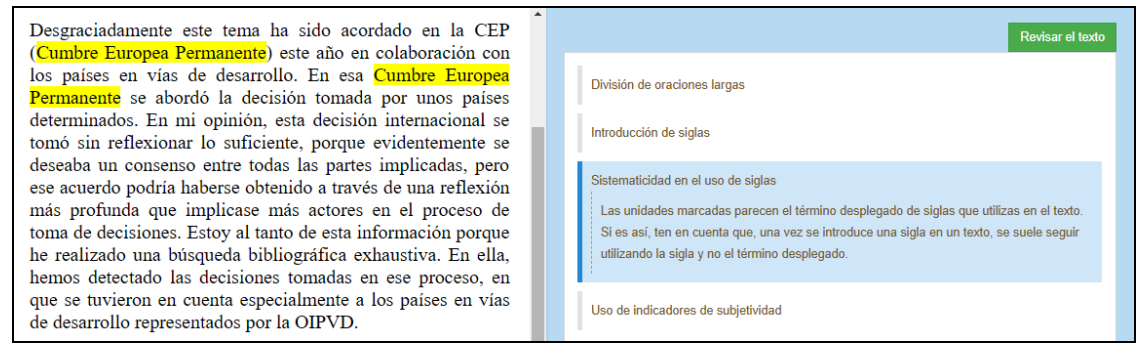

Figura 7. Detalle de una captura de pantalla de la herramienta que refleja la recomendación Sistematicidad en el uso de siglas 
d) "Uso de indicadores de subjetividad. Las unidades marcadas podrían ser indicadoras de subjetividad. Ten en cuenta que este tipo de textos suelen ser objetivos. Te recomendamos que revises estas unidades para confirmar que son adecuadas en tu texto."

En este caso, en el TFG del estudiante se detectan las unidades léxicas indicadoras de subjetividad. Para ello, la herramienta incluye un listado de este tipo de unidades, extraídas de Otaola (1988). Como puede observarse en la Figura 8, en el texto se marcan estas unidades, como desgraciadamente, en mi opinión y evidentemente, para que el estudiante decida si quiere eliminarlas de su escrito. Tal como se verá en el apartado 3.4, la herramienta integra un analizador morfosintáctico (Atserias et alii 2006), que permite lematizar automáticamente las diferentes unidades léxicas del texto y asignarles una categoría gramatical. Por tanto, en el caso del lema adjetival bueno, la herramienta lograría detectar las formas bueno, buena, buenos y buenas, en caso de aparecer en el texto.

\begin{tabular}{|l|l|}
\hline $\begin{array}{l}\text { Desgraciadamente este tema ha sido acordado en la CEP } \\
\text { (Cumbre Europea Permanente) este año en colaboración con }\end{array}$ \\
$\begin{array}{l}\text { los paises en vías de desarrollo. En esa Cumbre Europea } \\
\text { Permanente se abordó la decisión tomada por unos países } \\
\text { determinados. En mi opinión, esta decisión internacional se } \\
\text { tomó sin reflexionar lo suficiente, porque evidentemente se } \\
\text { deseaba un consenso entre todas las partes implicadas, pero } \\
\text { ese acuerdo podría haberse obtenido a través de una reflexión } \\
\text { más profunda que implicase más actores en el proceso de } \\
\text { toma de decisiones. Estoy al tanto de esta información porque } \\
\text { he realizado una búsqueda bibliográfica exhaustiva. En ella, } \\
\text { hemos detectado las decisiones tomadas en ese proceso, en } \\
\text { que se tuvieron en cuenta especialmente a los países en vías }\end{array}$ \\
de desarrollo representados por la OIPVD.
\end{tabular}

Figura 8. Detalle de una captura de pantalla de la herramienta que refleja la recomendación Uso de indicadores de subjetividad

e) "Variación de conectores. Los conectores de la lista siguiente se repiten varias veces en el texto. Haz clic en cada conector para ver sugerencias de conectores alternativos."

Esta recomendación muestra en la columna derecha una lista de los conectores discursivos que se repiten en el TFG tres o más veces. Si el estudiante hace clic en alguno de ellos, verá en el texto marcadas sus ocurrencias y, además, obtendrá en la misma columna derecha un listado de conectores alternativos (tanto intraoracionales como interoracionales) que expresan la misma relación discursiva. Por ejemplo, en la Figura 9 se marcan en el texto cuatro ocurrencias del conector de reformulación es decir, y en la columna derecha se muestra una lista con cuatro propuestas de conectores alternativos, como son dicho de otro modo, en otras palabras, esto es y o sea. 


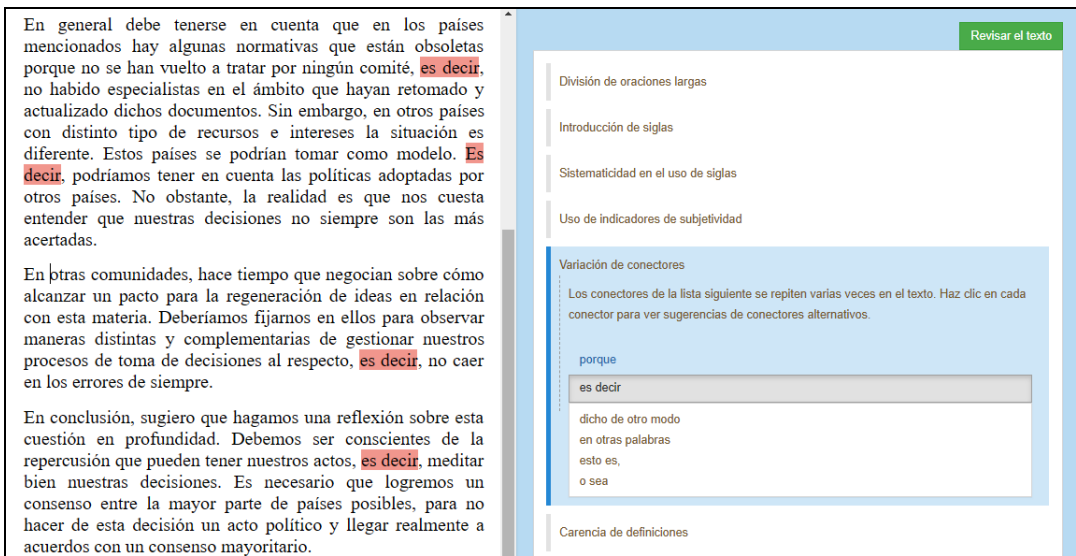

Figura 9. Detalle de una captura de pantalla de la herramienta que refleja la recomendación Variación de conectores

f) "Carencia de definiciones. Parece que no has incluido ninguna definición en el texto. Ten en cuenta que en este tipo de textos suelen incluirse definiciones."

En este caso, la herramienta revisa el TFG en busca de definiciones, ya que se presupone que un trabajo de este tipo suele incluirlas, especialmente en algunos apartados, como Marco teórico o Estado de la Cuestión. Para detectar las definiciones, la herramienta utiliza tres patrones definitorios básicos extraídos de Alarcón (2009): concebir como, definir como y denominar. En caso de que no se encuentren definiciones en el texto, la herramienta lo indica en la columna derecha y sugiere añadir alguna. En la Figura 10, siguiendo con el mismo texto incluido en las figuras anteriores, se muestra un ejemplo en que no hay definiciones en el texto y donde se visualiza la recomendación sobre esta cuestión.

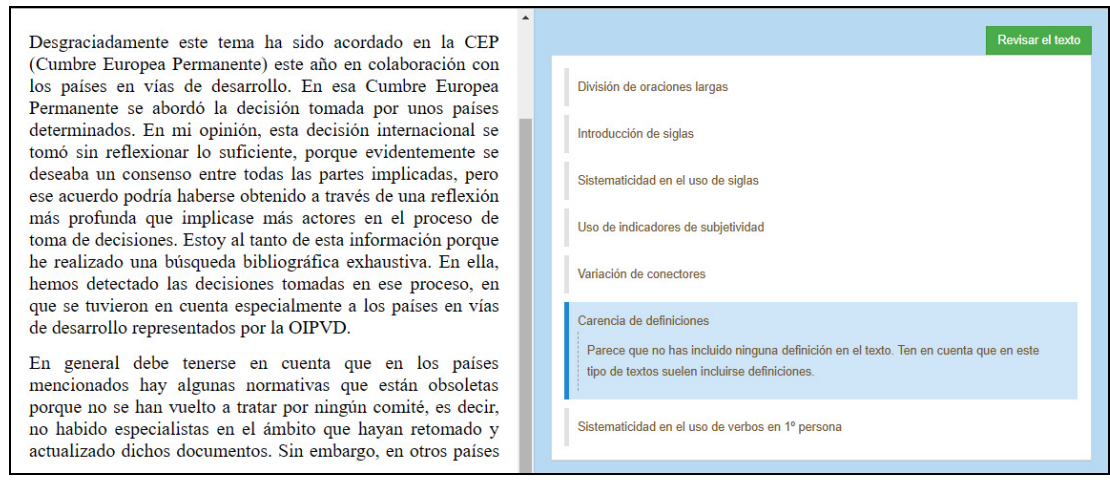

Figura 10. Detalle de una captura de pantalla de la herramienta que refleja la recomendación Carencia de definiciones 
g) "Sistematicidad en el uso de verbos en $1^{a}$ persona. Las unidades marcadas en verde parecen verbos en $1^{\mathrm{a}}$ persona del singular y las marcadas en azul parecen verbos en $1^{\mathrm{a}}$ persona del plural. Te recomendamos que optes por el singular o el plural para que el texto sea sistemático."

Esta recomendación tiene que ver con la falta de sistematicidad en el uso de la $1^{\text {a }}$ persona del singular y del plural, especialmente en relación con el autor del escrito. Hay ocasiones en que en un texto es necesario utilizar ambas formas, pero, en un TFG, es importante que el autor utilice el singular o el plural de manera sistemática (además de las formas impersonales, muy recomendables en este tipo de textos). Así, la herramienta marca en el texto en verde las formas verbales en singular y en azul las formas en plural, dando así información al estudiante para tomar la decisión de hacer modificaciones en este sentido. Por ejemplo, en la Figura 11, se marcan en verde las formas en singular he y sugiero, mientras que se destacan en azul las formas hemos, podríamos, deberíamos, hagamos, debemos y logremos.

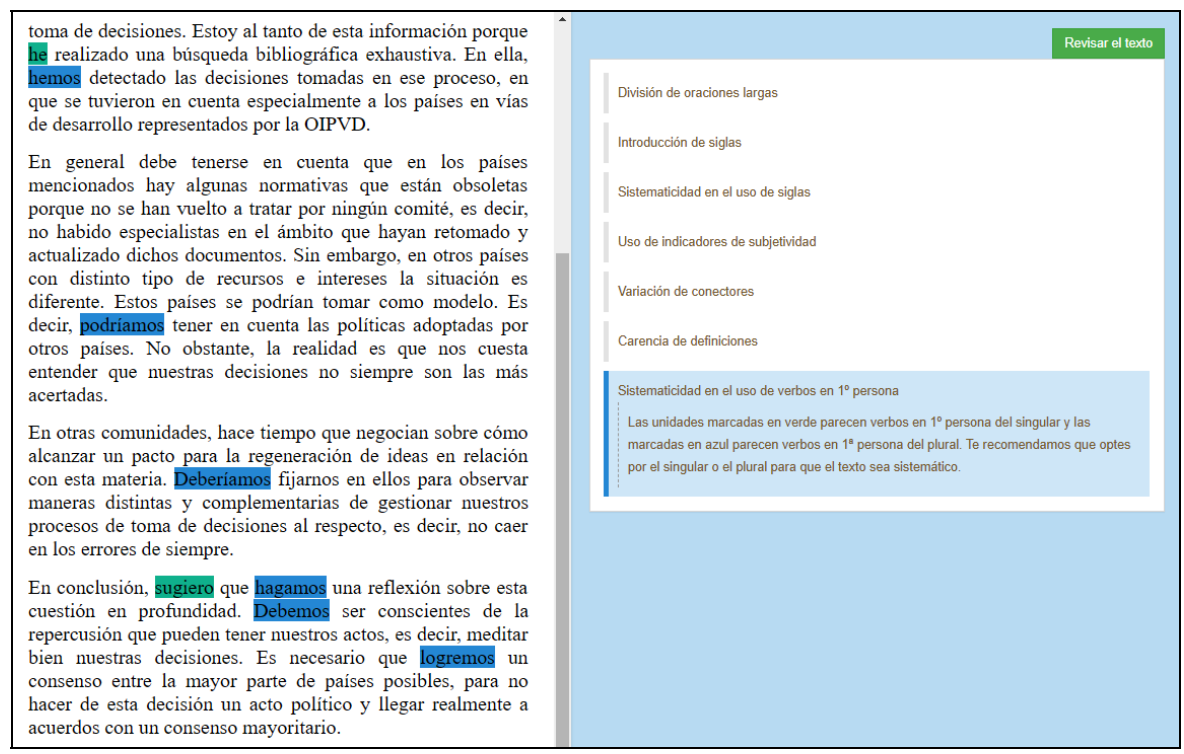

Figura 11. Detalle de una captura de pantalla de la herramienta que refleja la recomendación Sistematicidad en el uso de verbos en $1^{a}$ persona

\subsection{Recursos empleados para la implementación de la herramienta}

En cuanto a las especificaciones técnicas de la implementación, la herramienta fue desarrollada en un entorno LINUX usando un servidor APACHE y una base de datos MySQL. Se utilizaron también diferentes recursos tanto en el back-end (BASH, PERL y PHP, con un entorno de trabajo Laravel) como en el front-end (HTML, CSS, JAVSCRIPT, con AJAX y JQUERY). La herramienta está optimizada para su utilización con el navegador Google Chrome. 
Para su implementación se integraron, por un lado, dos herramientas de PLN existentes actualmente para el español, que ya se avanzaron en el apartado 3.3, y que permiten realizar un procesamiento lingüístico del texto escrito por el estudiante. En primer lugar, se incorporó el analizador morfosintáctico de Freeling (Atserias et alii 2006), mediante el cual se lematizan todas las unidades léxicas del texto y se asigna una categoría gramatical a cada una de ellas. En segundo lugar, se integró un segmentador discursivo (da Cunha et alii 2012b), que permite dividir el texto en oraciones y, además, en segmentos discursivos intraoracionales, siguiendo el concepto de segmento discursivo de Tofiloski et alii (2009). Por otro lado, la herramienta contiene dos listas de unidades léxicas, también mencionadas en el apartado 3.3. La primera lista incluye conectores interoracionales e intraoracionales que evidencian las 8 relaciones discursivas utilizadas en este trabajo. La segunda lista recoge unidades léxicas que indican subjetividad, como superlativos (ej. buenísimo), y ciertos adjetivos (ej. bueno), adverbios (ej. evidentemente) y frases (ej. sin ninguna duda).

En cuanto a la exportación e importación de documentos, por una cuestión de protección de datos, se decidió que la herramienta no guardase en su servidor los textos escritos por los estudiantes. Por tanto, si el estudiante desea guardar un texto escrito en línea con la herramienta, debe hacerlo en local. Para ello, existen varias opciones de exportación de documentos. Para poder importar un texto posteriormente en la herramienta debe utilizarse un formato que se ha creado específicamente para ello. Los detalles sobre la exportación e importación de documentos se recogen en el manual de uso de la herramienta (véase Nota 17).

\section{EVALUACIÓN DE LA HERRAMIENTA}

Para conocer la percepción de la utilidad de la herramienta por parte de estudiantes universitarios, se realizó una evaluación user-driven (basada en usuarios). Especialmente nos interesaba la valoración del Módulo 3 de la herramienta, para determinar si las recomendaciones incluidas son realmente útiles para la revisión de los TFG. Para ello, se contó con 77 estudiantes de la asignatura Habilidades de comunicación oral y escrita de $1^{\circ}$ del Grado en Educación Primaria de la Universidad Autónoma de Madrid (UAM). Aunque lógicamente el TFG se realiza en el último curso del grado, en esta evaluación seleccionamos estudiantes de $1^{\circ}$ porque queríamos conocer la percepción de estudiantes recién ingresados en la universidad, y también motivarlos de cara a la redacción del futuro TFG que tendrán que realizar. Concretamente, se les pidió que revisaran las funcionalidades de la herramienta en línea, que escribieran un texto en el editor (específicamente, el apartado Marco teórico de un hipotético TFG, donde suele ser necesaria la argumentación, la definición de conceptos y teorías, la incorporación de siglas, etc.) y que revisasen su texto con la herramienta automática, aplicando las recomendaciones de mejora que considerasen de utilidad. Una vez hecho esto, se les envió un cuestionario de valoración, elaborado con la herramienta Google Forms, en que se incluyeron preguntas de respuesta cerrada divididas en cuatro bloques:

1. Valoración general del módulo de revisión del texto.

2. Valoración de las recomendaciones.

3. Motivación hacia la escritura.

4. Valoración global de la herramienta. 
En general, los participantes hicieron una valoración muy positiva de la herramienta. La valoración global final indica que el 95\% de los estudiantes usaría la herramienta en futuros trabajos académicos, el 91\% la utilizaría para escribir su TFG y el 96\% la recomendaría a otros estudiantes. Estos resultados, del Bloque 4 (Valoración global de la herramienta), se ilustran en la Figura 12 en forma de gráficos. ${ }^{20}$ En este caso, las respuestas posibles eran Sí o No.

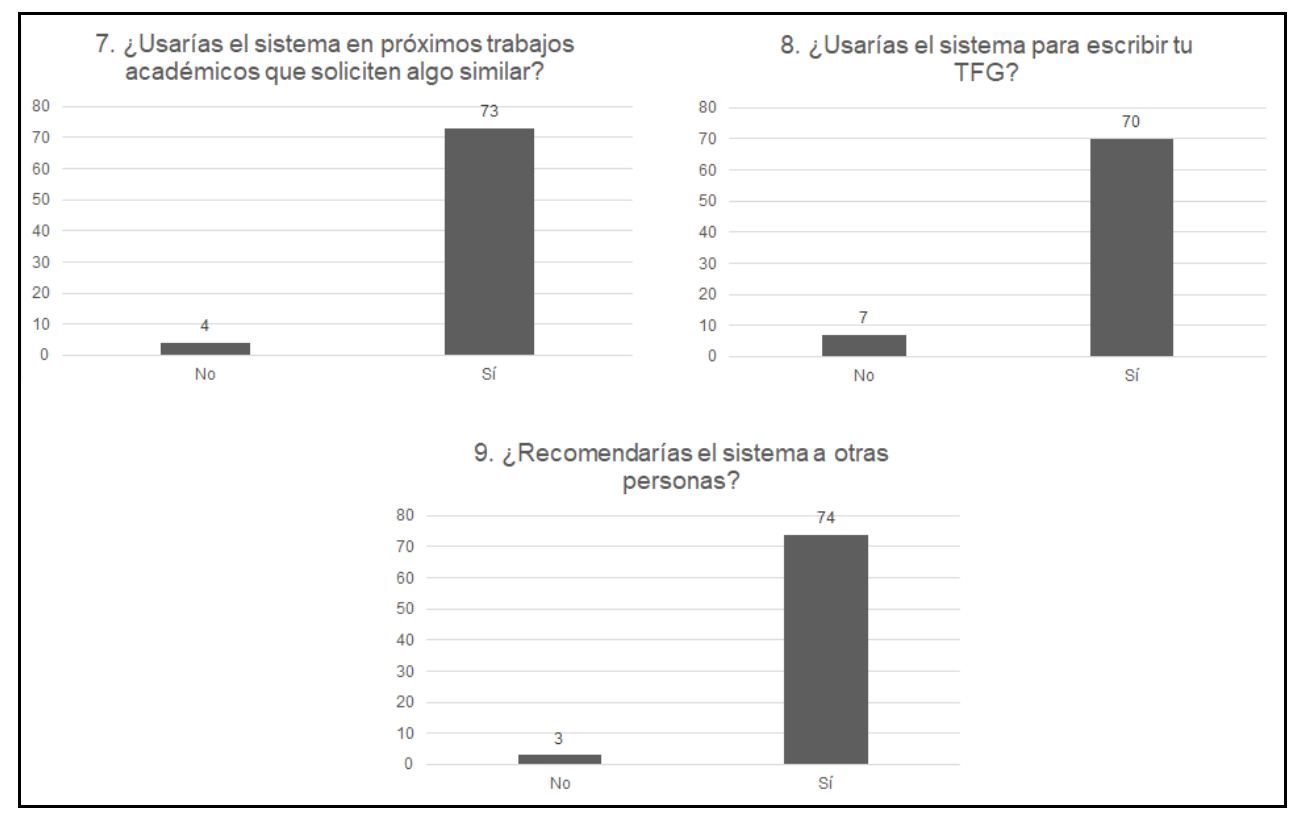

Figura 12. Resultados de las preguntas del Bloque 4 del cuestionario de valoración de la herramienta

Por su parte, el contenido de las recomendaciones obtenidas de la revisión lingüística del texto fue bien valorado en general, puesto que el $81 \%$ de los estudiantes las consideró útiles (concretamente, un 36\% las consideró muy útiles y un $45 \%$ bastante útiles). La redacción de las recomendaciones también se valoró positivamente, aunque con un resultado ligeramente inferior, puesto que un $78 \%$ de estudiantes consideró que eran claras (en este caso, un 22\% consideró que eran muy claras y un $56 \%$ bastante claras). Estos resultados, del Bloque 1 (Valoración general del módulo de revisión del texto), se ilustran en la Figura 13. En este caso, las respuestas posibles eran Mucho, Bastante, Poco y Nada.

20 En la parte superior de los gráficos mostrados en este apartado se indica la pregunta realizada, en la parte inferior las diferentes opciones de respuesta y encima de cada columna el número absoluto de estudiantes que seleccionaron cada opción de respuesta. 


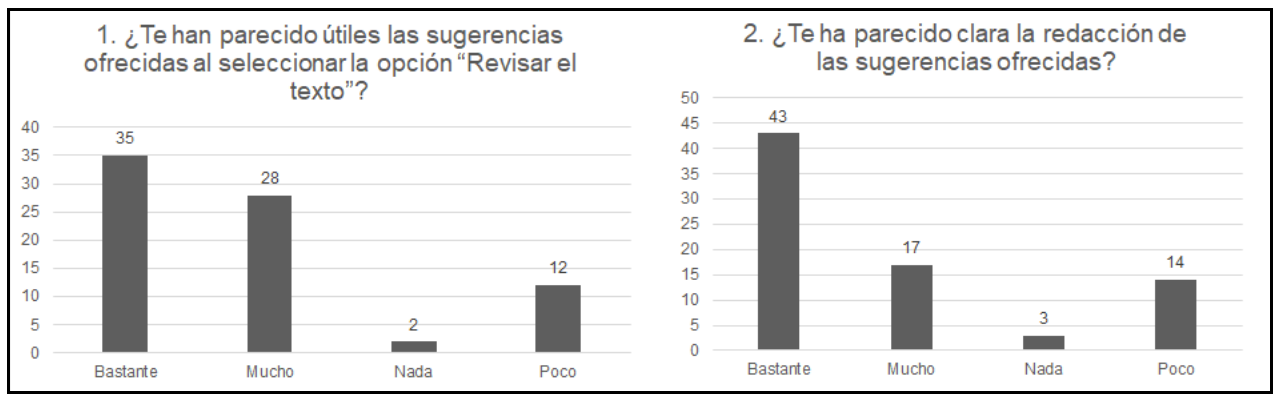

Figura 13. Resultados de las preguntas del Bloque 1 del cuestionario de valoración de la herramienta

En cuanto a la valoración de cada una de las recomendaciones, se obtuvieron diversos resultados interesantes. Estos resultados, del Bloque 2 (Valoración de las recomendaciones), se ilustran en la Figura 14. En este caso, las opciones de respuesta eran las siguientes:

- 0 = No he usado esta opción.

- $1=$ Nada.

- $2=$ Poco.

- 3 = Bastante.

- 4 = Mucho.

Tal como se observa en la Figura 14, las recomendaciones mejor valoradas involucran el uso de conectores discursivos. Por ejemplo, el $51 \%{ }^{21}$ consideró que la recomendación Variación de conectores era útil (concretamente, un 39\% consideró que era muy útil y un $12 \%$ bastante útil). Esto refleja que cada estudiante tiene tendencia a utilizar los mismos conectores en el texto para expresar determinadas relaciones discursivas y que esta recomendación les ayuda a emplear conectores alternativos que aporten riqueza léxica a su escrito. La recomendación División de oraciones largas también fue bien valorada, puesto que un $56 \%$ de estudiantes la consideró de utilidad (un $31 \%$ muy útil y un $27 \%$ bastante útil). Sin embargo, en este caso también hubo un $31 \%$ que la consideró poco útil. Por tanto, parece que los resultados dependen de la complejidad del texto escrito por el estudiante, pero que, en general, sí les resulta útil esta recomendación para lograr concisión en el texto. Cuanto más elaboradas y complejas sean las oraciones (y por tanto involucren el uso de conectores discursivos), más posibilidad hay de que esta recomendación sea ofrecida por la herramienta y resulte pertinente para el estudiante. A partir de estos resultados, parece que se consolida la idea de que los estudiantes universitarios tienen carencias sobre el tema de los conectores discursivos y valoran positivamente el tener herramientas de mejora en este sentido para utilizar en la redacción de sus TFG.

21 En las preguntas del Bloque 2, algunos estudiantes dejaron alguna pregunta en blanco o introdujeron un valor incorrecto. Por este motivo, los porcentajes de este bloque se han calculado sobre el total de estudiantes que respondió cada pregunta correctamente, que son 75 o 76 en función de la pregunta, en lugar del total de 77 estudiantes que respondieron las preguntas de los otros tres bloques. 


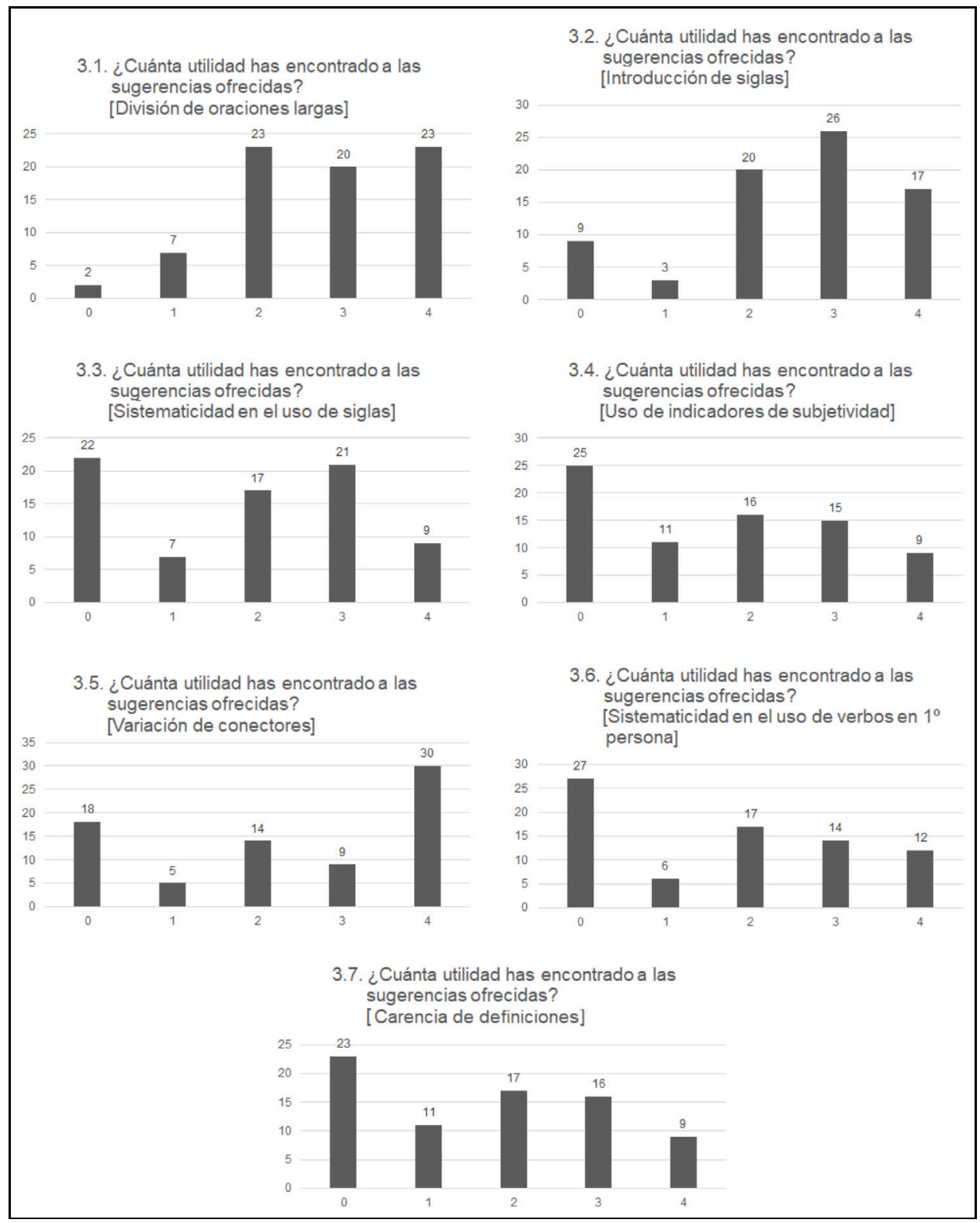

Figura 14. Resultados de las preguntas del Bloque 2 del cuestionario de valoración de la herramienta 
En relación con las siglas, la recomendación Introducción de siglas es una de las mejor valoradas, ya que un $58 \%$ de estudiantes la consideró útil (un $23 \%$ muy útil y un $35 \%$ bastante útil), aunque es cierto también que un $27 \%$ la valoró como poco útil. En este caso entra en juego el número de siglas que el estudiante haya insertado en su texto. Los resultados reflejan, así, que más de la mitad de los estudiantes ha tenido problemas con esta cuestión y ha olvidado en alguna ocasión añadir el término desplegado a su correspondiente sigla la primera vez que esta aparece en el texto. En el caso de la recomendación Sistematicidad en el uso de siglas, por su parte, llama la atención el 29\% de estudiantes que indica no haber utilizado esta recomendación. Esto quiere decir que la herramienta no les ha ofrecido recomendaciones sobre esta cuestión, es decir, que estos estudiantes fueron sistemáticos a la hora de utilizar las siglas en sus textos (después de haber introducido previamente tanto la sigla como el término desplegado la primera vez que aparecían en el texto). De todas maneras, también es cierto que un $40 \%$ considera útil la recomendación (un $12 \%$ muy útil y un $28 \%$ bastante útil). Se concluye, por tanto, que los estudiantes consideran interesantes las recomendaciones que les ayuden a lograr sistematicidad en sus TFG a la hora de incorporar este tipo de unidades léxicas.

Las recomendaciones para las que hubo resultados más heterogéneos fueron Uso de indicadores de subjetividad, Carencia de definiciones y Sistematicidad en el uso de verbos en $1^{a}$ persona. En estos casos, llama la atención el elevado porcentaje de estudiantes a quienes la herramienta no ha ofrecido esta recomendación (33\%, 30\% y 36\%, respectivamente). Esto quiere decir que estos estudiantes en particular no insertaron en sus textos unidades léxicas subjetivas de la lista incluida en la herramienta (lo cual es correcto, porque en este tipo de textos debe tenderse hacia la objetividad), sí insertaron definiciones (lo cual es adecuado en un TFG, especialmente en el apartado Marco teórico), y no tuvieron problemas de sistematicidad entre la $1^{a}$ persona del singular y del plural (seleccionando, por tanto, el singular o el plural para marcar de manera consistente el autor del TFG). El resto de estudiantes valoró estas tres recomendaciones de manera muy dispar, marcando de manera similar las diferentes opciones (Nada, Poco, Bastante y Mucho), como puede verse en la Figura 14. Esto quiere decir que también hubo estudiantes que encontraron dificultades en relación con estas cuestiones.

Finalmente, en cuanto a motivación hacia la escritura a través de la práctica con la herramienta en línea propuesta, se observa una valoración muy positiva por parte de los estudiantes. Un $89 \%$ de ellos indicó que las recomendaciones le ayudaron a mejorar su texto (el 38\% indicó que le ayudaron mucho y el 51\% bastante). Un 92\% consideró que las recomendaciones ofrecidas contribuyen a que aprenda a escribir mejor (el $45 \%$ consideró que contribuyen mucho y el $47 \%$ bastante). Al $81 \%$ le resultó motivadora la tarea de revisión del texto mediante la herramienta (al 32\% le resultó muy motivadora y al $49 \%$ bastante). Se consolida así la idea de que los estudiantes universitarios valoran positivamente la utilización de herramientas tecnológicas que les ayuden a escribir sus TFG. Estos resultados, del Bloque 3 (Motivación hacia la escritura), se ilustran en la Figura 15. En este caso, las respuestas posibles eran Mucho, Bastante, Poco y Nada, para las tres preguntas. 


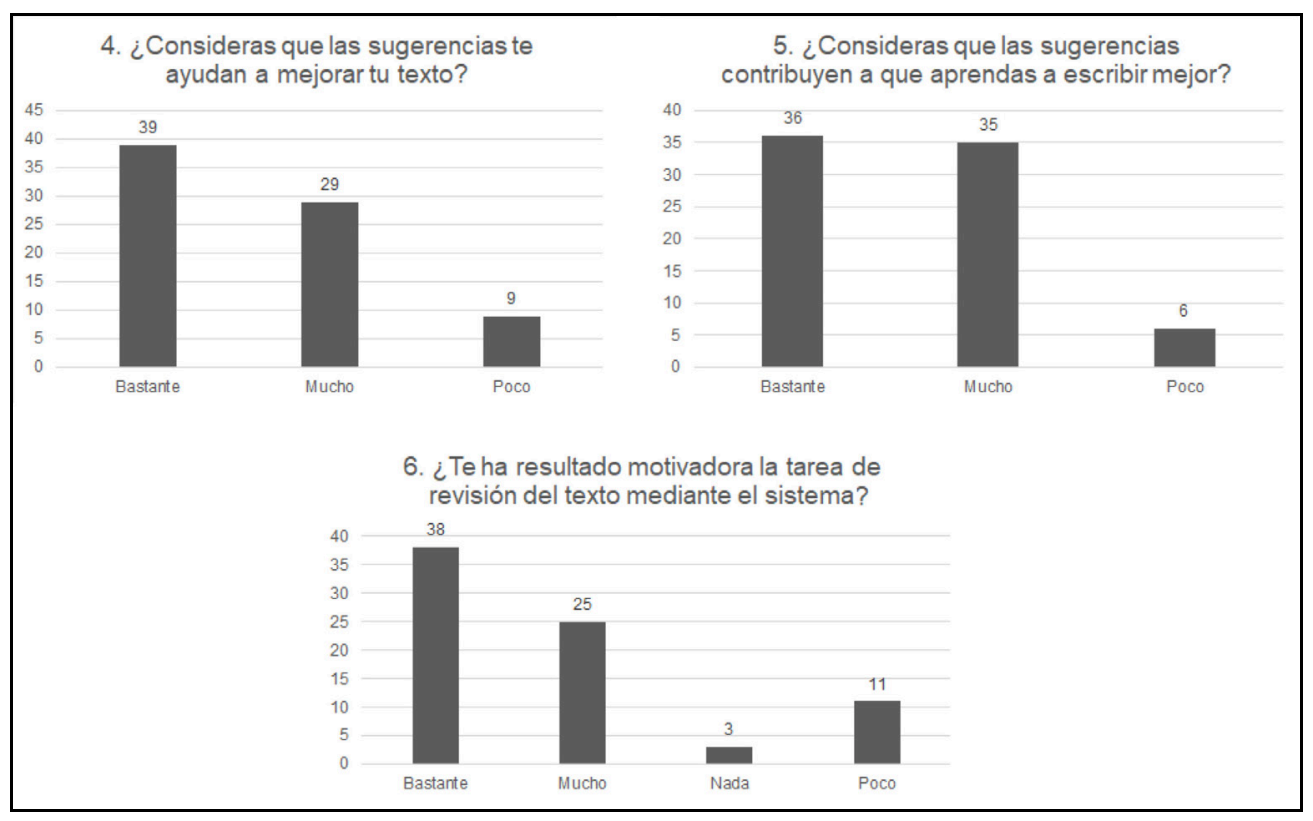

Figura 15. Resultados de las preguntas del Bloque 3 del cuestionario de valoración de la herramienta

\section{CONCLUSIONES Y TRABAJO FUTURO}

Este trabajo parte de la necesidad que tienen los estudiantes de las diferentes universidades españolas de elaborar el género textual TFG en el marco del Plan Bolonia. Aunque cada vez hay más publicaciones y cursos específicos para orientarles (por ejemplo, sobre las etapas de la elaboración del trabajo, la metodología de investigación o las cuestiones formales, como la citación bibliográfica), el desarrollo de herramientas tecnológicas puede suponer un complemento didáctico que aporte dinamismo al proceso de elaboración, redacción y revisión del TFG, especialmente a los estudiantes universitarios de hoy en día, que son nativos digitales. En este contexto, el objetivo de este trabajo ha sido, precisamente, diseñar e implementar una herramienta TIC para ayudar a este colectivo en la redacción del TFG en español, en la modalidad de trabajo de investigación.

La herramienta tiene forma de editor de textos en línea, integra recursos de PLN (un analizador morfológico y un segmentador discursivo), y está dividida en tres módulos, que ayudan al estudiante a: 1) estructurar el TFG, 2) darle formato y corregirlo ortográficamente, y 3) revisar la adecuación del texto, mediante recomendaciones lingüísticas específicas para este género textual, relacionadas con oraciones largas, conectores discursivos, siglas, definiciones, unidades léxicas subjetivas y rasgos morfosintácticos. El Módulo 1 es especialmente relevante porque no existía hasta la fecha ninguna herramienta tecnológica que ayudase al estudiante a estructurar su TFG, incorporando los apartados prototípicos de este género textual y añadiendo en cada uno de ellos los títulos, contenidos y fraseología habituales en 
este tipo de trabajos. El Módulo 3 es de utilidad sobre todo para revisar la adecuación del texto escrito por el estudiante, atendiendo a las características globales que debe tener este tipo de trabajos, como son la adecuación gramatical y estilística, la precisión, la concisión, la objetividad y la sistematicidad.

La evaluación user-driven realizada con estudiantes universitarios ofrece resultados positivos de la herramienta, ya que más del $90 \%$ indica que la usará en futuros trabajos académicos, que la utilizará para escribir su TFG y que la recomendaría a otros estudiantes. Con respecto a la productividad de las recomendaciones lingüísticas ofrecidas en el Módulo 3 , destacan especialmente las relacionadas con el uso de conectores discursivos. Se afianza así la idea de que los estudiantes universitarios tienen carencias sobre esta cuestión (Villar et alii 2018) y este trabajo contribuye aportando estrategias novedosas que involucran el uso de conectores, de cara a que la redacción de los estudiantes mejore (Errázuriz 2012, 2014). Los resultados indican también que las recomendaciones sobre cuestiones relacionadas con la sistematicidad del uso de las siglas son pertinentes y útiles para los estudiantes universitarios.

Este trabajo tiene una transferencia directa de resultados a la sociedad en diversos aspectos. Teniendo en cuenta que esta herramienta TIC está en línea y que cualquier persona puede utilizarla gratuitamente, podría ser considerada como una herramienta de e-learning para los estudiantes universitarios, llegado el momento de realizar su TFG o incluso otro tipo de trabajos académicos, ya que el Módulo 3 podría emplearse para revisar cualquier escrito del ámbito universitario. Asimismo, puede ser una herramienta didáctica interesante para que los profesores universitarios expliquen el proceso de elaboración de un TFG en el aula de manera interactiva, puesto que la utilización de este tipo de recursos motiva a los estudiantes, tal como reflejan los resultados de esta investigación.

Como trabajo futuro, y teniendo en cuenta los resultados de la evaluación realizada, en primer lugar, se refinarán las recomendaciones para que logren una mayor cobertura, es decir, para que detecten un mayor número de casos. Para ello se ampliará el concepto de sigla y de definición (como se ha mencionado en el apartado 3, actualmente la herramienta únicamente reconoce siglas propias y tres patrones definitorios). También se ampliará el listado de relaciones discursivas, y se ofrecerán sus correspondientes conectores intraoracionales e interoracionales. En segundo lugar, se investigará sobre la posibilidad de incluir nuevas recomendaciones no contempladas por el momento. Finalmente, en cuanto a la evaluación, sería interesante realizar un experimento similar pero con estudiantes que ya estén elaborando el TFG, es decir, estén en $4^{\circ}$ de grado, para observar si los problemas de redacción que tienen son los mismos o si, por el contrario, su paso por la universidad les ha hecho mejorar sus destrezas en el ámbito de la escritura académica.

\section{AGRADECIMIENTOS}

Este trabajo se ha llevado a cabo en el marco de un contrato Ramón y Cajal (RYC-201416935) financiado por el Ministerio de Economía, Industria y Competitividad, vinculado al Departamento de Filologías Extranjeras y sus Lingüísticas de la Facultad de Filología de la Universidad Nacional de Educación a Distancia (UNED). Los resultados se derivan de dos proyectos de investigación. Por un lado, del proyecto "Un sistema automático de ayuda a la redacción de textos especializados de ámbitos relevantes en la sociedad española actual”, fi- 
nanciado en la Convocatoria 2015 de Ayudas Fundación BBVA a Investigadores y Creadores Culturales (IN[15]_HMS_LIN_0371). Por otro lado, del proyecto "Tecnologías de la Información y la Comunicación para la e-Administración: hacia la mejora de la comunicación entre Administración y ciudadanía a través del lenguaje claro (TIC-eADMIN)", financiado por el Ministerio de Ciencia, Innovación e Universidades en la convocatoria 2018 de Proyectos I+D del Subprograma Estatal de Generación de Conocimiento (PGC2018-099694-A-I00). Ambos proyectos se han desarrollado en el marco del grupo de investigación ACTUALing de la UNED, en colaboración con el grupo de investigación IULATERM del Institut de Lingüística Aplicada (IULA) de la Universitat Pompeu Fabra (UPF). Quiero agradecer su participación en la investigación a todos los miembros del equipo de trabajo de ambos proyectos, sobre todo a M. Amor Montané y a Luis Hysa. Agradezco especialmente a Juan Antonio Núñez, coordinador del Centro de Escritura de la Facultad de Formación de Profesorado y Educación de la UAM, su apoyo en relación con el cuestionario de valoración de la herramienta.

\section{REFERENCIAS BIBLIOGRÁFICAS}

Alarcón, R. 2009. Descripción y Evaluación de un Sistema Basado en Reglas para la Extracción Automática de Contextos Definitorios. Barcelona: IULA, Universitat Pompeu Fabra. Tesis doctoral.

Alcaraz, E., J. Mateo y F. Yus (coords.) (2006). Las lenguas profesionales y académicas. Barcelona: Ariel.

Amat, O. y A. Rocafort (coords.) (2017). Cómo investigar: Trabajo fin de grado, tesis de máster, tesis y otros proyectos de investigación. Barcelona: Profit.

Anduiza, E. y M. Méndez (en línea). Guía práctica 1. Elaboración de trabajos. Murcia: Área de Ciencia Política y de la Administración, Universidad de Murcia. http://www. um.es/cpaum/files/recursos/1-F4cc9345d11288254557-rec-1-1.pdf (13-01-2020).

J. Atserias, B. Casas, E. Comelles, M. González, Ll. Padró y M. Padró (2006). "Freeling 1.3. syntactic and semantic service in an open-source NLP library". En Proceedings of the 5th International Conference on Language Resources and Evaluation (LREC 2006). pp. 48-55.

Barrios, M. y J. Barrientos (eds.) (2016). El trabajo de fin de grado. Teorías y prácticas. Madrid: Vision Libros.

Botta, M. (2002). Tesis, monografías e informes: nuevas normas y técnicas de investigación y redacción. Buenos Aires: Biblos.

Briz, A., S. Pons y J. Portolés (coords.) (2008). Diccionario de partículas discursivas del español. www.dpde.es (13-01-2020)

Cabré, M. T. (1993). La Terminología. Teoría, Métodos, Aplicaciones. Barcelona: Antártida.

Cabré, M. T. (1999). La Terminología: Representación y comunicación. Elementos para una Teoría de Base Comunicativa y Otros Artículos. Serie Monografías 3. Barcelona: IULA, Universitat Pompeu Fabra.

Cabré, M. T. (2016). "Prólogo. Redactar un proyecto, una capacidad adquirida". En El trabajo de fin de grado y de máster. Redacción, defensa y publicación. Barcelona: Editorial UOC, pp. 11-14.

Carlino, P. (2005). Escribir, leer y aprender en la universidad: una introducción a la alfabetización académica. Buenos Aires: Fondo de Cultura Económica. 
Cassany, D. (2005). La cocina de la escritura. Barcelona: Anagrama.

Cassany, D. (2006). Taller de textos: Leer, escribir y comentar en el aula. Barcelona: Paidós.

Cassany, D. (2007). Afilar el lapicero. Guía de redacción para profesionales. Barcelona: Anagrama.

Castelló, M. (2007a). (coord.). Escribir y comunicarse en contextos científicos y académicos. Conocimientos y estrategias. Barcelona: Graó.

Castelló, M. (2007b). "El proceso de composición de textos académicos". En Castelló, M. (coord.). Escribir y comunicarse en contextos científicos y académicos. Conocimientos y estrategias. Barcelona: Graó, pp. 47-82.

Castelló, M. (2014). "Los retos actuales de la alfabetización académica: estado de la cuestión y últimas investigaciones”. En Ballano, I. e I. Muñoz (eds.). La escritura académica en las universidades españolas. Bilbao: Universidad de Deusto, pp. 153-176.

Castelló, M., M. Mateos, N. Castells, A. Iñesta, I. Cuevas e I. Solé (2012). “Academic writing practices in Spanish universities". Electronic Journal of Research in Educational Psychology, 10(2), pp. 569-590.

Cierlica, P. (2018). Cómo hacer tu trabajo de fin de grado: Guía paso a paso para escribir tu TFG con éxito. Amazon.

Codina, L1. (en línea). Trabajos académicos. https://www.lluiscodina.com/culturaacademica/tesis-doctorales/ (13-01-2020).

Conroy, M. (2010). "Internet tools for language learning: University students taking control of their writing". Australasian Journal of Educational Technology, 26(6), pp. 861-882.

Corral, C. (2010). Los conectores discursivos de la lengua escrita en la clase de español como lengua extranjera: una propuesta de trabajo. León: Universidad de León. Tesis doctoral.

Cuenca, M. J. (2006). La connexió i els connectors. Perspectiva oracional i textual. Vic: Eumo.

da Cunha, I., E. SanJuan, J-M. Torres-Moreno, M. T. Cabré y G. Sierra (2012a). “A Symbolic Approach for Automatic Detection of Nuclearity and Rhetorical Relations among Intrasentence Discourse Segments in Spanish". Lecture Notes in Computer Science, 7181, pp. 462-474.

da Cunha, I., E. SanJuan, J-M. Torres-Moreno, I. Castellón y M. Lloberes (2012b). "DiSeg 1.0: The first system for Spanish discourse segmentation". Expert Systems With Applications, 39(2), pp. 1671-1678.

da Cunha, I. (2016). El trabajo de fin de grado y de máster. Redacción, defensa y publicación. Barcelona: Editorial UOC.

Errázuriz, M. C. (2012). “Análisis del uso de los marcadores discursivos en argumentaciones escritas por estudiantes universitarios". Perfiles educativos, 34(136), pp. 98-117.

Errázuriz, M. C. (2014). "El desarrollo de la escritura argumentativa académica: los marcadores discursivos". ONOMÁZEIN, 30, pp. 217-236.

Ferrer, V. R., M. Carmona y V. Soria (2013). El trabajo de fin de grado: guía para estudiantes, docentes y agentes colaboradores. Madrid: McGraw-Hill.

García, M. P. y P. Martínez (coords.) (2012). Guía práctica para la realización de trabajos fin de grado y trabajos fin de máster. Murcia: Universidad de Murcia, Servicio de Publicaciones. 
Gamallo P., M. García, I. del Río e I. González (2015). “Avalingua: Natural Language Processing for Automatic Error Detection”. En Callies, M. y S. Götz (eds.). Learner Corpora in Language Testing and Assessment. Ámsterdam: John Benjamins, pp. 35-58.

Giraldo, J. J. (2008). Análisis y descripción de las siglas en el discurso especializado de genoma humano y medio ambiente. Barcelona: Institut Universitari de Lingüística Aplicada.

González García, J. M., A. León y M. Peñalba (2014). Cómo escribir un trabajo de fin de Grado: algunas experiencias y consejos prácticos. Madrid: Síntesis.

Icart, M. T., C. Fuentelsaz y A. M. Pulpón (2001). Elaboración y presentación de un proyecto de investigación y una tesina. Barcelona: Publicacions de la Universitat de Barcelona.

Iruskieta, M. e I. da Cunha (2010). "Marcadores y relaciones discursivas en el ámbito médico: un estudio en español y euskera". En Bueno, J.L. (coord.). Analizar datos > Describir variación, Analysing data > Describing variation. Vigo: Universidade de Vigo, Servizo de Publicacións, pp. 146-159.

Jakobson, R. (1963). Essais de linguistique genérale. París: Minuit.

Jiménez-Yáñez, R. M. (2020). Comunicar en la Universidad y en la vida profesional. Pamplona: EUNSA, Ediciones Universidad de Navarra.

Kruse, O. (2003). "Getting started: Academic writing in the first year of a university education”. En Björk, L., G. Bräuer, L. Rienecker y P. Stray Jörgensen (eds.). Teaching academic writing in European higher education. Netherlands: Springer, pp. 19-28.

López Escarcena, S. (2011). "Para escribir una tesis jurídica: técnicas de investigación en derecho". Ius et Praxis, 17(1), pp. 231-246.

Mann, W. C. y S. A. Thompson (1988). Rhetorical structure theory: Toward a functional theory of text organization. Text, 8(3), pp. 243-281.

Mari, J. A. (1998). Manual de redacción cientifica. Ediciones digitales. http:// edicionesdigitales.info/Manual/manual.pdf (13-01-2020).

Martínez, J. (2012). Descripción y variación retórico-funcional del género Tesis Doctoral: Un análisis desde dos disciplinas y dos comunidades discursivas a partir del Corpus Te DiCE-2010. Valparaíso: Pontificia Universidad Católica de Valparaíso. Tesis doctoral.

Martínez, J. (2015). "El género Tesis Doctoral de Historia y Física: Descripción y variación retórico-funcional". En Parodi, G. y G. Burdiles (eds.). Leer y escribir en contextos académicos y profesionales: Géneros, Corpus y Métodos. Valparaíso: Ediciones Universitarias de Valparaíso, pp. 113-152.

Martínez, C. y C. Llamas (2015). "Implantación del Centro de Escritura en la Universidad de Navarra: líneas generales y pruebas de diagnóstico”. En Ballano, I. e I. Muñoz (eds.). La escritura académica en las universidades españolas. Bilbao: Universidad de Deusto, pp. 11-40.

Meza, P. e I. da Cunha (2019). "Comunicación del conocimiento propio y relaciones discursivas en el género tesis". Sintagma, 31, pp. 103-130.

Meza, P. (2013). La comunicación del conocimiento en las secciones de Tesis de Lingüística: determinación de la variación entre grados académicos. Valparaíso: Pontificia Universidad Católica de Valparaíso. Tesis doctoral.

Meza, P. (2015). "La comunicación del conocimiento en el género Tesis de Lingüística: Comparación entre grados académicos.” En Parodi, G. y G. Burdiles (eds.). Leer y 
escribir en contextos académicos y profesionales: Géneros, Corpus y Métodos. Valparaíso: Ediciones Universitarias de Valparaíso, pp. 67-112.

Montolío, E. (2001). Conectores de la lengua escrita. Barcelona: Ariel.

Montolío, E. (2014). Manual de escritura académica y profesional. Barcelona: Ariel.

Núñez (coord.) (2015). Escritura académica: De la teoría a la práctica. Madrid: Pirámide.

Otaola, C. (1988). "La modalidad (con especial referencia a la lengua española)". Revista de Filología Española, 68(1), pp. 97-117.

Pantoja, A. (coord.) (2009). Manual básico para la realización de tesinas, tesis y trabajos de investigación. Madrid: EOS.

Parodi, G. (coord.) (2008). Géneros académicos y géneros profesionales. Accesos discursivos para saber y hacer. Valparaíso: Pontificia Universidad Católica de Valparaíso.

Parodi, G. (ed.) (2010). Academic and professional discourse genres in Spanish. Ámsterdam: John Benjamins.

Parodi, G. y G. Burdiles (2015). Leer y Escribir en contextos académicos y profesionales: géneros, corpus y métodos. Santiago de Chile: Ariel.

Portolés, J. (1998). Marcadores del discurso. Barcelona: Ariel.

Rigo, A. y G. Genescà (2002). Cómo presentar una tesis y trabajos de investigación. Barcelona: Octaedro.

Rodríguez-Piñeiro, A. I. (coord.) (2014). Guía para la elaboración de trabajos académicos de investigación. Cádiz: Universidad de Cádiz.

Sabino, C. (2003). Cómo hacer una Tesis y elaborar todo tipo de escritos. Bogotá: Panamericana.

Sánchez Herrera, J. (en línea). Trabajos académicos y cientificos: sugerencias de realización. Las fases de elaboración de un trabajo académico. Tenerife: Departamento de Sociología, Universidad de La Laguna. http://fjsher.webs.ull.es/materiales/apoyo_ Sugerencias_Trabajos.pdf (13-01-2020).

Sánchez-Jiménez, D. (2016). "Implicaciones de la citación en la voz del autor en el discurso académico universitario: la memoria de máster escrita en español por estudiantes españoles y filipinos". Diálogo de la Lengua, 8, pp. 16-36.

Sánchez-Jiménez, D. (2018). "Reseña de El trabajo de fin de grado y de máster. Redacción, defensa y publicación", Círculo de Lingüística Aplicada a la Comunicación, 76, pp. 303-308.

Santos Río, L. (2003). Diccionario de partículas. Salamanca: Luso-Española.

Savio, A. (2010). "Las huellas del autor en el discurso académico: un estudio sobre tesis de psicoanalistas argentinos". Lenguaje, 38(2), pp. 563-590.

Swales, J. M. (1990). Genre Analysis: English in Academic and Research Settings. Cambridge: Cambridge University Press.

Tapia, M. y G. Burdiles (2012). "La Organización Retórica del Marco Referencial en Tesis de Trabajo Social”. Alpha (Osorno), 35, pp. 169-184.

Tolchinsky, L., A. Escofet y M. J. Rubio (2003). Tesis, tesinas y otras tesituras. De la pregunta de investigación a la defensa de la tesis. Barcelona: Edicions de la Universitat de Barcelona.

Tofiloski, M., J. Brooke y M. Taboada (2009). "A syntactic and lexical-based discourse segmenter". En Proceedings of the 47th Annual Meeting of Association for Computational Linguistics. Singapur: Association for Computational Linguistics, pp. 77-80. 
Tortosa, V. (2014). Metodología de la investigación cientifica. Guía para la elaboración del trabajo académico humanístico. Alicante: Universidad de Alicante.

Eco, U. (1997). Cómo se hace una Tesis. Técnicas y procedimientos de investigación, estudio y escritura. Barcelona: Editorial Gedisa.

van Dijk, T.A. (1977). Text and Context. London: Longman.

van Dijk, T.A. (1989). La ciencia del texto. Paidós: Barcelona.

Venegas, R., M. T. Núñez, S. Zamora y A. Santana (2015). Escribir desde la pedagogía del género. Guías para Escribir el Trabajo Final de Grado en Licenciatura. Valparaíso: Pontificia Universidad Católica de Valparaíso.

Villar, E. M., A. Fuerte, J. Vertiz, E. Gálvez y J. A. Arévalo (2018). “Actividades colaborativas en el aprendizaje de marcadores discursivos en estudiantes universitarios". Propósitos y Representaciones, 6(2), pp. 607-618.

Walker, M. (2000). Cómo escribir trabajos de investigación. Barcelona: Editorial Gedisa. 